\title{
An energy residual-based approach to gradient effects within the mechanics of generalized continua
}

\begin{abstract}
Generalized continua exhibiting gradient effects are addressed through a method grounded on the energy residual (ER)-based gradient theory by the first author and coworkers. A main tool of this theory is the ClausiusDuhem inequality cast in a form differing from the classical one only by a nonstandard extra term, the (nonlocality) ER, required to satisfy the insulation condition (its global value has to vanish or to take a known value). The ER carries in the nonlocality features of the mechanical problem through a strain-like rate field, being the specific nonlocality source, and a concomitant higher-order long-range stress (or microstress) field. The thermodynamic restrictions on the constitutive equations are determined by the latter inequality with no need for microstress equilibrium equations, whereas the principle of virtual power (PVP) is left in a standard format. The derived state equations include a set of partial differential equations involving the nonlocality-source strain-like quantity and the related long-range stress, as well as the associated higher-order boundary conditions determined by the insulation condition. Second-grade materials within gradient elasticity, gradient plasticity and crystal plasticity, as well as materials with microstructure (micromorphic and Cosserat materials) are considered to derive the pertinent constitutive equations. The proposed ER-based approach to gradient effects is shown to constitute a more straightforward and "economic" way to formulate the relevant constitutive equations than the PVP-based one.
\end{abstract}

Keywords: Cosserat continua; generalized continua; gradient elasticity; gradient plasticity; nonlocal continuum thermodynamics.

\footnotetext{
*Corresponding authors: Castrenze Polizzotto, Dipartimento di Ingegneria Civile Ambientale Aerospaziale e Materiali, Università di Palermo, Viale delle Scienze 90128, Palermo, Italia Aurora A. Pisano, Dipartimento Patrimonio Architettonico e Urbanistico, Università Mediterranea di Reggio Calabria, via Melissari 89124, Reggio Calabria, Italia, e-mail: castrenze.polizzotto@unipa.it; aurora.pisano@unirc.it
}

\section{Introduction}

The main concern of this paper is the treatment of generalized continua within the framework of strain gradient theories. For generalized continua, here we intend the (nonpolar) gradient continua addressed by Mindlin and Tiersten [1], Toupin [2, 3], Mindlin [4] and Mindlin and Eshel [5], the (polar) Cosserat materials [6-8], the micromorphic continua (or continua with a microstructure) studied by Eringen and Suhubi [9], Mindlin [10] and the like. Conceived as elastic materials capable by their own nature of accounting for some microstructural effects as typically size effects, they were soon extended to elastoplasticity within the framework of internal variables [7, 11-14]. Aifantis [15-18] first envisioned the gradients of strain as extra state variables rendering the strain gradient models suitable to capture size effects of various types, like for instance strengthening effects, and strain localization phenomena. The pioneering work by Aifantis opened the route to a plethora of gradient theories that made it possible to predict and describe several experimentally detected phenomena, e.g., various types of size effects [19-21], strain localization [22, 23] and interface interaction effects [24-27]. For a review of the inherent literature, see, e.g., Fleck and Hutchinson [28], Aifantis [29], Kuroda and Tvergaard [30].

Generalized continua viewed as gradient models were addressed by Germain [31, 32] by means of the principle of virtual power (PVP) suitably extended such as to incorporate the additional intrinsic working modes of the material and the additional degrees of freedom of the continuum. This made it possible to envisage the inherent microstresses and to assess the related field and boundary equilibrium equations. This technique was also used by Maugin [13, 33] and Maugin and Muschik [34] in conjunction with continuum thermodynamics in order to establish the constitutive equations for material models with internal variables. A general elastic-thermoplastic framework for generalized continua has been recently provided by Forest and Sievert [8], Forest [35] and Forest and Aifantis [36], Forest et al. [37] in the form of a "micromorphic 
approach”. This approach constitutes a generalization of a number of gradient plasticity theories existing in the literature [e.g., Gao et al. [38], Acharya and Bassani [39], Fleck and Hutchinson [40], Liebe and Steinmann [42], Gurtin [42-45], Gudmundson [46], Anand et al. [47], Fleck and Willis [48, 49], Voyiadjis and Deliktas [50], Voyiadjis et al. [51], and others]. All the latter theories have in common the use of the mentioned extended PVP; for this reason, we will refer to them (including the micromorphic approach) as PVP-based theory or theories.

A common feature of the PVP-based theories is to operate within a local-type framework through a procedure consisting in two steps. These, with a terminology widely used within the latter theories, can be described as follows:

1. Application of the extended PVP to obtain, besides the standard equilibrium equations, the analogous equations for the inherent microstresses including the related microtraction boundary conditions (microstress equilibrium equations).

2. Application of the Clausius-Duhem inequality in its classical (local) form to deduce the relevant restrictions on the constitutive equations, to be used as a basis for the subsequent formulation of the evolution-diffusion laws satisfying the second thermodynamics principle.

Step 2 provides the state equations of the microstresses, which are algebraic in nature, such as for classical simple materials. The latter equations are transformed into a set of partial differential equations (PDEs) with the associated boundary conditions (which indeed is the typical format of the constitutive equations for gradient materials) as soon as they are combined with the microstress equilibrium equations of step 1 .

However, it may be desirable to envision an alternative procedure in which step 2, powered by a suitable (weak nonlocal) Clausius-Duhem inequality, is able to provide directly the mentioned differential-form constitutive equations and the related boundary conditions, whereas (a weakened) step 1 makes use of just the standard (i.e., non-extended) PVP. Although such a procedure implies exiting out of the local-type framework and thus abandoning the classical thermodynamics principle of the local actions, it is of interest because, first, the PVP is left in its standard format and, second, the number of state variables to consider is reduced and ultimately the procedure simplifies without loss in generality.

The energy residual (ER)-based gradient theory (hereafter referred to as ER-based theory), advanced by the first author and coworkers for gradient plasticity [27, 52-59], gradient elasticity [53] and gradient damage [60], happens to conform to the above nonlocal-type framework. The central point of the mentioned theory consists in an extra scalar state variable, called (nonlocality) $E R$, say $P$, which represents the long-range energy density transmitted to the generic particle of the body by the surrounding material. $P$ is a macroscopic energy measure of the complex interaction phenomena occurring at the microscale; it is representable in the typical form [58]:

$$
P=\nabla \cdot \mathbf{g}, \mathbf{g}=\mathbf{B}: \dot{\varepsilon}^{\mathrm{nl}}=\left\{B_{i j k} \dot{\varepsilon}_{j k}^{\mathrm{nl}}\right\}
$$

where the vector $\mathbf{g}$ is the long-range energy flux (similar to, but physically different from, the heat flux) and the symbol $\dot{\boldsymbol{\varepsilon}}^{\text {nl }}$ denotes a strain-like rate tensor (or vector, or even scalar) quantity constituting the source of nonlocality, whereas $\mathbf{B}$ denotes a long-range stress-like tensor of order one unit more than $\dot{\varepsilon}^{\mathrm{nl}}$, to which it is associated. $P$ intervenes into the energy balance equation (first thermodynamics principle, written in pointwise form) as an extra energy term carrying in the nonlocality features of the material behavior.

Another featuring point of the ER-based theory is the so-called insulation condition [52, 58, 61] expressing the physical concept that the total amount of long-range energy flux supplied to the whole body has to vanish or to be equal to a known value. This permits one to enforce, in a straightforward manner, some (higher-order) boundary conditions interpreting the physical influence of the exterior ambient onto the material microstructure.

The above ER-based theory has been developed over a period of as long as approximately 15 years with improvements along the way. It is applicable not only to gradient materials [see Polizzotto [58] for the most progressed form of it as a gradient theory] but also to true nonlocal (integral) materials [62, 63]. As a gradient theory, it exhibits many similarities with the PVP-based theory. Comparisons have been accomplished in several papers $[27,56,58,59]$ pointing out that the two theories although operating within different (nonlocal/local) theoretical frameworks - lead ultimately to substantially the same results, but the ER-based theory reaches the goal in a more direct and "economic" way. The purpose of the present paper is to make a further step in the development of the ER-based theory by addressing generalized continua.

It is useful to mention here that the roots of the ERbased theory go back to Edelen and Laws [61] (see also Eringen and Edelen [64]), where the concept of "nonlocality residual" was advanced as the energy supplied to the generic material point at the expense of the surrounding 
material. Whereas the global energy balance equation is there enforced in its classical format, its pointwise form instead includes the latter nonlocality residual as an additional scalar term, whose integral over the whole domain is postulated to vanish.

Aifantis [15, 17], referring to monodimensional materials with microstructure whose macroscopic behavior is described by means of internal variables, advanced the seminal idea of accounting for their inherent nonlocal nature by introducing a dependence of the free energy on the gradient of the internal variable(s) and postulated an energy balance equation enriched by an extra energy term. This term, quite similar to our ER $P$, was heuristically cast in various forms and in particular as the divergence of a vector linearly dependent on the internal variable rate(s), i.e., a vector like our long-range energy flux $\mathbf{g}$ of (1.1).

A concept similar to the mentioned "long-range energy flux" is that of "interstitial work flux" advanced by Dunn and Serrin [65] in a study oriented toward Korteweg-type materials, i.e., capillary fluids endowed with an internal energy function depending on the material density and its gradient, among others. With a rigorous thermodynamic reasoning, the above interstitial work flux was found (at least for a wide subclass of materials) to possess the form of a product between a third-order stress tensor and the time rate of the nonlocality-source strain rate, namely, a form like (1.1) ${ }_{2}$. A more general class of capillary fluids was addressed by Casal and Gouin [66] by means of an internal energy function depending on the density, the entropy and their gradients. As there are two different nonlocality sources, these authors considered two different vector fields, the sum of which is comparable to the long-range energy flux in (1.1). The standard pressure and temperature are replaced with more general entities of analogous meaning, but the second thermodynamics principle remains unaltered formally.

Maugin [13] (see also Maugin and Muschik [34]) addressed elastic-plastic and elastic-viscoplastic materials with internal variables in the hypothesis that the free energy function depends on the elastic strain, the temperature, a scalar internal variable and its gradient. Following Müller [67], the former quoted authors advanced the concept of "extra entropy flux" as a vector field added to the actual entropy flux. This means that the gradient effects are accounted for through the thermal laws of the material, even in the case of a nonlocality source being of a purely mechanical nature. The extra entropy flux is formally like our long-range energy flux; however, the latter flux variable, in contrast to the extra entropy flux, does not involve the temperature whenever the nonlocality source is of mechanical nature, whereas the entropy flux and the heat equation remain always unaltered, even in the case of a nonlocality source originating from the temperature or the entropy.

The paper is organized as follows. In Section 2, the ER-based gradient theory is presented together with some basic concepts of thermodynamics, on which it is grounded, and a comparison between the latter theory and the analogous PVP-based one is also given. Section 3 is devoted to second-grade materials within gradient elasticity with particular attention to the Aifantis model [23, 29]. In Section 4, second-grade plastically gradient materials are addressed, first by the ER approach, second by the PVP one, for a detailed comparison; single crystal gradient plasticity is also addressed. Section 5 is devoted to micromorphic materials, both elastic and elastic-plastic, as well as to Cosserat materials with strengthening effects. Conclusions are drawn in Section 6.

\subsection{Notation}

As a rule a compact notation is used with boldface letters for vectors and tensors of any order. Index contraction is denoted by as many dots as the number of contracted index pairs. Considering $\mathbf{a}=\left\{a_{i}\right\}, \mathbf{B}=\left\{B_{i j}\right\}, \mathbf{C}=\left\{C_{i j k}\right\}$ and $\mathbf{D}=\left\{D_{i j k h}\right\}$, we can write $\mathbf{a} \cdot \mathbf{B}=\left\{a_{i} B_{i j}\right\}, \mathbf{B}: \mathbf{C}=\left\{B_{i j} C_{i j k}\right\}, \mathbf{D}: \mathbf{B}=\left\{D_{i j k h-}\right.$ $\left.B_{k h}\right\}, \mathbf{a} \otimes \mathbf{B}=\left\{a_{i} B_{j k}\right\}, \mathbf{D}:(\mathbf{a} \otimes \mathbf{C})=\left\{D_{i j \mathrm{kh}} a_{j} C_{k h}\right\}$, where $\otimes$ denotes tensor product, and the summation rule for repeated indices holds. Orthogonal Cartesian coordinates are used throughout, say $\mathbf{x}=\left(x_{1}, x_{2}, x_{3}\right)$. The symbol $\nabla=\left\{\partial_{i}\right\}$ denotes the gradient operator, $\nabla \mathbf{B}=\left\{\partial_{i} B_{j k}\right\}$. An upper dot denotes time derivative, say $\dot{A}=\partial A / \partial t$. The symbol:= means equality by definition; $(\cdot)^{T}$ is the transpose of $(\cdot)$. The Ricci alternating third-order tensor is denoted by $\mathbf{e}=\left\{e_{i j k}\right\}$. Other symbols will be defined in the text at their first appearance.

\section{Fundamentals}

In the following, "material system" means a continuous collection of particles with its specific deformation modes, each mode being described by a strain-like variable; it transforms into a "continuum system" (or "body", or even "structural system") when these deformation modes are required to comply with the compatibility conditions expressed in terms of displacements, $\mathbf{u}$, or more in general of degrees of freedom. The material system is the natural ambient to discuss questions related to the constitutive behavior. The term "nonlocal", often used in the 
following, is as a rule intended as "weak nonlocal" except whenever differently stated. In the following, we assume that the material occupies a finite (three-dimensional) domain $V$ of boundary surface $S=\partial V$.

According to a widely accepted definition by Noll [68], a material is called simple material whenever the stress state at a point $\mathbf{x}$ and time $t$ depends solely on the strain state at $\mathbf{x}$ measured by a standard (second-order symmetric) strain tensor and evaluated through its actual value at $t$, as well as its past history up to $t$. Here we extend the latter definition by introducing the concept of local-type material, which is like in all a simple material except that it is featured by a richer deformation state described by means of both standard and higher-order strain tensors. Obviously, a simple material is local type, but the inverse does not hold generally.

\subsection{The ER-based gradient theory and related thermodynamic aspects}

A featuring starting point of the ER-based theory is the choice of a bilinear form to represent the pertinent (internal) stress power, say $\mathcal{W}[31,32]$. The most general form we can envision for it in the present context can be cast as follows:

$$
\mathcal{W}=\mathcal{W}_{u}+\mathcal{W}_{\xi}
$$

where

$$
\mathcal{W}_{u}:=\sigma: \dot{\varepsilon}(\mathbf{u})+\tau: \dot{\eta}(\mathbf{u}), \mathcal{W}_{\xi}:=\boldsymbol{s}: \dot{\chi}(\xi)+\mu: \dot{\boldsymbol{\kappa}}(\xi) .
$$

Here, $\varepsilon$ and $\eta$ are strain tensors of second and third order, respectively, which represent all the kinematically independent deformation modes of the continuum, controlled by the primary degrees of freedom of the structural system, i.e., the displacements $\mathbf{u}$. Similarly, $\chi$ and $\boldsymbol{\kappa}$, are some microstrain tensors that represent the analogous kinematically independent deformation modes of the material microstructure and are controlled by the secondary degrees of freedom, say $\xi$, (like, typically, the local rotation of a Cosserat continuum or the microstrain of a micromorphic material). The higher-order tensor $\boldsymbol{\kappa}$, is of order one unit more than $\chi$; the latter is here formally treated as a second-order tensor, although in practice it may also be a vector, or even a scalar.

For kinematically independent deformation modes here we mean those tensor-valued deformation measures to which the material is reactive, but are kinematically distinct from one another; elastic and plastic strains do constitute just one deformation mode of the material. An additive decomposition rule holds for the strains $\varepsilon$ and $\eta$ into elastic and plastic parts, namely,

$$
\varepsilon=\varepsilon^{\mathbf{e}^{\mathrm{e}}}+\varepsilon^{\mathrm{P}}, \eta=\eta^{\mathrm{e}}+\eta^{\mathfrak{p}}
$$

An analogous decomposition can be stated for $\chi$ and $\kappa$, in the case of dissipative microstructure $[8,35,36]$.

The symbols $\sigma, \tau, \boldsymbol{s}, \boldsymbol{\mu}$ denote stress tensors workconjugate of $\dot{\varepsilon}, \dot{\eta}, \dot{\chi}, \dot{\kappa}$, respectively. They possess the common features of contributing to the formation of the stress power $\mathcal{W}$ and of being linked - through the associated strain tensors - to the (either primary or secondary) degrees of freedom, $\mathbf{u}$ and $\boldsymbol{\xi}$.

The stress power $\mathcal{W}$ is required to be an objective quantity, i.e., invariant under change of the observer [31, 32]. This implies that $\mathcal{W}=0$ whenever the deformation modes are all associated to a rigid-body motion of the form

$$
\mathbf{u}(\mathbf{x}, t) \rightarrow \mathbf{u}^{\star}(\mathbf{x}, t)=\mathbf{d}(t)+\mathbf{W}(t) \cdot \mathbf{x}
$$

where $\mathbf{d}(t)$ is a vector and $\mathbf{W}(t)$ a second-order skewsymmetric tensor, both varying with time. As the microstructure moves like the continuum during such a transformation, the way $\chi$ and $\kappa$, change correspondingly is straightforward, but depends on their particular physical meaning (it thus needs to be specified for every particular case).

A second featuring point of the ER-based theory is the choice of the internal energy potential, say $e$, together with the set of state variables on which it depends. It is here cast in the form:

$$
e=e(\varepsilon, \eta, \chi, \kappa ; \nabla \varepsilon, \nabla \chi ; \gamma, \nabla \boldsymbol{\gamma} ; p, N) .
$$

Here, the first group of arguments, i.e., $\varepsilon, \eta, \chi, \kappa$, includes what we have called "kinematically independent deformation modes", each being controlled by either the primary or the secondary degrees of freedom. The latter arguments constitute observable strain variables and do intervene in the formation of the stress power $\mathcal{W}$ of (2.1) and (2.2). The second group of arguments includes the gradients $(\nabla \varepsilon, \nabla \chi)$ of the lower-order observable strain variables $(\varepsilon, \chi)$. These gradient variables are mutually exclusive with the respective higher-order observable strain variables $(\eta, \kappa)$. In other words, whenever a gradient variable (either $\nabla \varepsilon, \nabla \chi$, or both) is actually present as an argument of $e$, then the corresponding higher-order strain variable is assumed to be lacking and the stress power $\mathcal{W}$ loses the related contribution; on the contrary, if a higherorder strain variable (either $\boldsymbol{\eta}, \boldsymbol{\kappa}$, or both) is actually an argument of $e$, then the corresponding gradient variable is assumed not to appear as an argument of $e$.

The third group of arguments $(\gamma, \nabla \gamma)$ constitutes a particular category of internal variables here called 
pseudo-internal variables. These behave like true internal variables, as they control the changes of the constitutive behavior of the material and do not contribute to the stress power. However, they cannot be qualified as true internal variables because the variable $\gamma$ is required to play as a set of extra degrees of freedom (similar to the "internal degrees of freedom" devised by Maugin [69]), and additionally, a spatial differential integration with the aid of suitable boundary conditions is needed for its evaluation. The above makes the latter variable $\gamma$ exhibit the characters of observable variables, which is in contrast to the true internal variables of classical thermodynamics [68]. A typical example of pseudo-internal variables is the plastic strain within gradient plasticity. The variable $\gamma$ is here treated as a second-order strain tensor, but it may in practice be a vector or a scalar.

Finally, the fourth group of arguments of $e$ includes the (true) internal variable $p$ (considered as a scalar, but on occasion it may be a vector or a tensor) and the entropy $N$.

The presence of a gradient variable as an argument of e indicates that either a lower-order observable strain variable, a lower-order pseudo-internal variable, or both constitute a nonlocality-source strain for the material.

In place of the internal energy $e$ of (2.5), we can use the free energy $\psi=e-N \theta$, i.e., a function of the form:

$$
\psi=\psi(\varepsilon, \eta, \chi, \kappa ; \nabla \varepsilon, \nabla \chi ; \gamma, \nabla \gamma ; p, \theta)
$$

which contains the same arguments as $e$, but the entropy $N$ is replaced by the temperature $\theta$.

A third featuring point of the ER-based theory is the introduction of an ER, $P$, having the meaning of the longrange power density supplied to the generic particle by the surrounding material as a consequence of the microstructual interactions promoted by the material nonlocal nature. $P$ is an additional (scalar) state variable, which intervenes into the (pointwise) energy balance equation (first thermodynamics principle), namely,

$$
\dot{e}=\mathcal{W}+P-\nabla \cdot \mathbf{q}+h
$$

where $\mathbf{q}$ is the conduction heat flux and $h$ the radiation heat supply. The ER $P$ can be viewed as an "extra stress power" density supplied to the generic particle due to the long-range actions, in contrast to $\mathcal{W}$ being the standard stress power due to the short-range actions. $P$ is identically vanishing if and only if the material is local type (but not necessarily simple), which means that no nonlocality sources are involved therein (i.e., no gradient-type variables are included as arguments of either $e$ or $\psi$ ).
Introducing the entropy production, say $\dot{\Gamma}$, defined as usual $[67,69]$, i.e.,

$$
\dot{\Gamma}=\dot{N}+\nabla \cdot\left(\frac{\mathbf{q}}{\theta}\right)-\frac{h}{\theta} \geq 0,
$$

where $\theta$ is the absolute temperature, then the (nonlocal) Clausius-Duhem inequality can be written either in terms of internal energy $e$, i.e.,

$$
\theta \dot{\Gamma}=\mathcal{W}+P-\dot{e}+\theta \dot{N}-\frac{\mathbf{q}}{\theta} \cdot \nabla \theta \geq 0,
$$

or in terms of free energy $\psi$, namely,

$$
\theta \dot{\Gamma}=\mathcal{W}+P-\dot{\psi}+N \dot{\theta}-\frac{\mathbf{q}}{\theta} \cdot \nabla \theta \geq 0 .
$$

As the latter inequalities must be satisfied for any deformation mechanism and for any evolution law of the material, either (2.9) or (2.10) can be used to derive the thermodynamic restrictions on the constitutive equations. These include the state equations governing the reversible behavior of the material, as well as the dissipation inequality, to take into account the subsequent formulation of the evolution/diffusion laws. The mentioned state equations turn out to exhibit an algebraic form for those state variables that do not constitute nonlocality sources, whereas they take on the form of PDEs (with associated higher-order boundary conditions) for the state variables playing the role of nonlocality sources. This derivation is based on procedural techniques well consolidated in the literature [70-73], so it will be accomplished in the following sections with only the explanations believed necessary for the clarity of the presentation.

\subsection{More about the ER $P$}

A relation must exist between the ER, $P$, and every nonlocality source strain, as for instance the pseudo-internal variable $\gamma$. This relation essentially depends on the form of the strain gradient by which the nonlocality effects manifest themselves, i.e., either the first gradient or a higher-order gradient. Here, we first address the case of the first gradient, namely, the case in which the free energy depends on the first strain gradient $\nabla \gamma$, which also includes the dependence on a function of $\nabla \gamma$, like $\|\nabla \gamma\|^{2}$, or $\operatorname{curl} \gamma=\mathbf{e}: \nabla \gamma$.

To find out this relation, we can follow reasoning quite similar to one by Polizzotto [58] for an analogous analysis. For completeness and more clarity, we repeat it 
here for the specific case of the pseudo-internal variable $\gamma$. Assuming that $\gamma$ constitutes the only nonlocality source, let us first note that $P$ can be expressed in terms of $\dot{\gamma}$ and $\nabla \dot{\gamma}$ as the power expended correspondingly, i.e.,

$$
P=\mathbf{A}: \dot{\gamma}+\mathbf{B}: \nabla \dot{\gamma}
$$

Here, A and $\mathbf{B}$ denote stress quantities work-conjugate of $\dot{\gamma}$ and $\nabla \dot{\gamma}$, respectively. Let us note that the global ER within any subdomain $v \subseteq V$ of the material can be set in the form

$$
\int_{v} P \mathrm{~d} v=\int_{\partial v} \mathbf{m}: \dot{\gamma} \mathrm{d} a
$$

where $\mathrm{d} a$ denotes the area of a surface element, and the traction-like tensor $\mathbf{m}=\left\{\mathrm{m}_{i j}\right\}$ is a second-order tensor describing the long-range actions of the outer ambient on the boundary surface $\partial v$. Then, introducing (2.11) and applying the divergence theorem, (2.12) gives the equality

$$
\int_{v}(\mathbf{A}-\nabla \cdot \mathbf{B}): \dot{\gamma} \mathrm{d} v+\int_{\partial v}(\mathbf{n} \cdot \mathbf{B}-\mathbf{m}): \dot{\gamma} \mathrm{d} a=0 .
$$

This is an identity holding for any choice of the subdomain $v$ and for any choice of the $\dot{\gamma}$ field, with $\mathbf{A}$ and $\mathbf{B}$ (assumed) independent of $\dot{\gamma}$; hence, the following conditions must be satisfied:

$$
\mathbf{A}=\nabla \cdot \mathbf{B}, \mathbf{n} \cdot \mathbf{B}=\mathbf{m} .
$$

Substituting (2.14) into (2.11) shows that whenever the nonlocality effects manifest themselves via the first-order strain gradient the ER $P$ takes on an explicit constitutive form as in (1.1), i.e.,

$$
P=\nabla \cdot \mathbf{g}=\nabla \cdot(\mathbf{B}: \dot{\gamma}), \mathbf{g}:=\mathbf{B}: \dot{\gamma}
$$

This means that $P$ can be interpreted as an energy source emerging from a diffusion or conduction-like phenomenon described by the long-range energy flux vector, $\mathbf{g}=\mathbf{B}: \dot{\gamma}$, (similar to, but physically different from, the heat conduction flux $\mathbf{q}$ [65]), and thus

$$
\int_{v} P \mathrm{~d} v=\int_{\partial v} \mathbf{n} \cdot \mathbf{B}: \dot{\gamma} \mathrm{d} a
$$

Equation (2.15) gives an explicit representation for $P$ in terms of a nonlocality-source strain rate $\dot{\gamma}$ and an associated long-range stress tensor $\mathbf{B}$, the order of which is a unit more than the order of $\gamma$. The latter representation can be interpreted as a (weak) nonlocal representation of $P$ as an extra stress power (for it is meaningful only if $\mathbf{B}$ and $\dot{\gamma}$ are continuous fields), in contrast to (2.11), which instead is a local representation (it is locally meaningful even if $\nabla \dot{\gamma}$ is considered independent of $\dot{\gamma}$ ).
In the case of dependence of the free energy $\psi$ on the Laplacian $\Delta y$, following a reasoning similar to the one developed previously for $\nabla \gamma$, we can find:

$$
\mathbf{g}:=\mathbf{B}:(\nabla \dot{\gamma})^{T}-\nabla \mathbf{B}: \dot{\gamma}, P=\nabla \cdot \mathbf{g}=\mathbf{B}: \Delta \dot{\gamma}-\Delta \mathbf{B}: \dot{\gamma}
$$

where $\mathbf{B}$ is a stress tensor of the same order as $\gamma$. The case of the second gradient of a nonlocality-source strain can be treated in an analogous way [53].

We finally observe that the diffusion phenomenon to which the ER can be associated can take place only as far as the nonlocality effects manifest themselves as gradient effects. Indeed, for a true nonlocal (integraltype) material $[62,63]$, the ER $P$ can be shown to be associated to a radiation-like phenomenon whereby $P$ is similar to, but physically different from, the heat radiation supply $h$.

\subsection{Insulation condition and boundary conditions within the ER approach}

Equation (2.16) states that the total ER within any subdomain $v \subseteq V$ equals the total long-range energy flux entering through its boundary surface $\partial v$. For $v=V$ and assuming that no interface exists in $V$ with surface nonlocality sources, (2.16) becomes

$$
\int_{V} P \mathrm{~d} v=\int_{S} \mathbf{n} \cdot \mathbf{B}: \dot{\gamma} \mathrm{d} a
$$

where $S=\partial V$. The traction-like quantity $\mathbf{n} \cdot \mathbf{B}$ is the longrange energy flux entering $V$ through the unit surface of normal $\mathbf{n}$ per unit $\dot{\gamma}$; it represents the long-range actions on $S$ from the outside of $V$. As in general the material system is constitutively insulated, such actions are null, and thus, (2.18) reads (insulation condition):

$$
\int_{S} \mathbf{n} \cdot \mathbf{B}: \dot{\gamma} \mathrm{d} a=0
$$

For a material system not being constitutively insulated (for it is enclosed within a larger system that instead is constitutively insulated), as demonstrated in Polizzotto [58], the insulation condition can be restated by considering a boundary interface $S$ with a surface ER $P_{S}=-\mathbf{m}: \dot{\gamma}^{+}$, where $\mathbf{m}$ denotes the long-range energy flux released by the interface and entering $V$, whereas $\dot{\gamma}^{+}$ is the value of $\dot{\gamma}$ at points external to $S$ but very close to it. The insulation condition (2.19) now has to be enforced as

$$
P_{\text {tot }}=\int_{V} P \mathrm{~d} v+\int_{S} P_{S} \mathrm{~d} a=\int_{S}(\mathbf{n} \cdot \mathbf{B}-\mathbf{m}): \dot{\gamma} \mathrm{d} a=0
$$


where the continuity of $\dot{\gamma}$, i.e., $\dot{\gamma}=\dot{\gamma}^{+}$on $S$, has been used. In the following, we shall prefer to write (2.20) in the nonhomogeneous form:

$$
\int_{V} P \mathrm{~d} v=\int_{S} \mathbf{n} \cdot \mathbf{B}: \dot{\gamma} \mathrm{d} a=\int_{S} \mathbf{m}: \dot{\gamma} \mathrm{d} a,
$$

such that $\mathbf{m}$ can be interpreted as the long-range action on $S$ coming from the environment exterior to $V$. By (2.20) or (2.21), the following higher-order boundary conditions must be satisfied:

$$
\dot{\gamma}=\mathbf{0} \text { on } S_{\mathrm{h}}, \mathbf{n} \cdot \mathbf{B}=\mathbf{m} \text { on } S_{\mathrm{f}}
$$

where $S_{\mathrm{h}}$ and $S_{\mathrm{f}}$ are a partition of $S, S=S_{\mathrm{h}} \cup S_{\mathrm{f}}$, respectively called "hard" and "free" after a labeling largely used within gradient plasticity $[44,45,47]$.

\subsection{The PVP-based theory}

As noted previously, the PVP-based theory saves a local-type vision of deformation phenomena, like within the classical approach to continuum mechanics but at the cost of introducing a larger set of state variables than may be strictly necessary. In fact, it considers any strain-like state variable and its gradient as being independent of each other at the constitutive level and introduces a related extra stress power cast in an explicit local form. For instance, making reference to the material model described previously, characterized by the internal energy function (2.5), the PVP-based theory posits the internal energy $e$ in the form

$$
e=e(\varepsilon, \eta, \chi, \kappa ; \gamma, \zeta ; p, N)
$$

which is similar to (2.5), but the gradients $\nabla \varepsilon, \nabla \chi$ have disappeared, whereas $\nabla \gamma$ is replaced by a third-order tensor, say $\zeta$. This $\zeta$ variable, thought of as independent of $\gamma$ at the constitutive level, is instead related to $\gamma$ by the gradient relation $\zeta=\nabla \gamma$ at the global structural level. Moreover, the energy balance equation (2.7) is stated in the form

$$
\dot{e}=\mathcal{W}_{\mathrm{enl}}-\nabla \cdot \mathbf{q}+h
$$

in which no residual $P$ is incorporated and the enlarged stress power $\mathcal{W}_{\text {enl }}$ is given by

$$
\mathcal{W}_{\mathrm{enl}}=\mathcal{W}+\underbrace{\mathbf{A}: \dot{\gamma}+\mathbf{B}: \dot{\xi}}_{\text {extra stress power }},
$$

where $\mathbf{A}$ and $\mathbf{B}$ denote the microstresses work-conjugate of the pseudo-internal variables rates $\dot{\gamma}, \dot{\zeta}$, respectively. Indeed, with the PVP approach, all the strain variables (including the gradient ones) are considered as observable strain variables and all the stresses are treated as stress power contributors, including the microstresses A, B (which instead within the ER approach do not happen to be such contributors). The same transformation $\mathcal{W}+P \rightarrow \mathcal{W}_{\text {enl }}$ to pass from (2.7) to (2.24) has obviously to be adopted for the Clausius-Duhem inequality, either (2.9) or (2.10). On the basis of the compatibility relations expressed in terms of primary $(\mathbf{u})$, secondary $(\chi)$ and extra $(\gamma)$ degrees of freedom, i.e.,

$$
\boldsymbol{\varepsilon}=\operatorname{sym} \nabla \mathbf{u}, \boldsymbol{\eta}=\nabla \boldsymbol{\varepsilon}, \boldsymbol{\kappa}=\nabla \chi, \zeta=\nabla \gamma,
$$

we shall see later on in more detail that the extended PVP provides, together with the standard equilibrium equations (which indeed may be already known), the microstress and microtraction equilibrium equations, formally like (2.14). Then, the constitutive equations cast as a set of PDEs with associated boundary conditions are derived through the elimination of the redundant state variables, like $\mathbf{A}$ (which in fact do not intervene into the latter PDEs and boundary conditions).

The above will be better clarified in Section 4, where a particular plastically gradient material model will be addressed with both the ER-based and the PVP-based theories. We shall see that the ER approach constitutes in general a more direct and "economic" method than the PVP one for the formulation of the constitutive equations in the presence of gradient effects.

\section{Second-grade elastic materials}

In this section, a few second-grade elastic structural models, i.e., governed by displacement gradients up to the second order, similar to those studied by Toupin $[2,3]$ and Mindlin [4], are addressed assuming isothermal conditions. These materials can be cast within the present framework by assuming $\chi \equiv \varepsilon$ and $\kappa \equiv \eta$ (the microstructure deforms like the macrostructure); also, $\gamma \equiv \mathbf{0}, p \equiv 0$, such that the free energy (2.6), in the assumed isothermal conditions, can be cast in the simpler form

$$
\psi=\psi(\boldsymbol{\varepsilon}, \boldsymbol{\eta}) .
$$

Also, the stress power (2.1) can be simplified by writing:

$$
\mathcal{W}=\sigma: \dot{\varepsilon}+\tau: \dot{\eta},
$$

where $\varepsilon=\left\{\varepsilon_{i j}\right\}$ and $\boldsymbol{\eta}=\left\{\eta_{k i j}\right\}$ describe the kinematically independent deformation modes of the material, controlled by the displacement $\mathbf{u}$ through the compatibility relations

$$
\boldsymbol{\varepsilon}=\operatorname{sym} \nabla \mathbf{u}, \quad \eta=\nabla \boldsymbol{\varepsilon},
$$


holding in the whole domain $V$. The stress tensors $\sigma$ and $\tau$ exhibit symmetry features like $\varepsilon$, i.e., with respect to the index pair $(i, j)$. With these choices, the stress power (3.2) proves to be invariant under change of the observer, as in fact any rigid-body motion of the type (2.4) gives $\dot{\varepsilon}\left(\mathbf{u}^{\star}\right)=\mathbf{0}, \dot{\eta}\left(\mathbf{u}^{*}\right)=\mathbf{0}$.

\subsection{Local-type elastic material}

Let us assume that the considered nonsimple material is a local-type elastic model characterized by the free energy (3.1) and the stress power (3.2). The higher-order strain $\eta$ is independent of $\varepsilon$ at the constitutive level; hence, $\varepsilon$ does not play the role of nonlocality-source strain, and thus the Clausius-Duhem inequality (2.10) takes on the form:

$$
D=\sigma: \dot{\varepsilon}+\tau: \dot{\eta}-\dot{\psi} \geq 0,
$$

where $D=: \theta \dot{\Gamma}$ denotes the intrinsic dissipation density. No ER has to appear in (3.4); hence the ER approach and the PVP one collapse into the same procedure. On expanding the time derivative of $\psi$, from the latter inequality we easily obtain the pertinent elasticity laws, namely

$$
\boldsymbol{\sigma}=\frac{\partial \psi}{\partial \boldsymbol{\varepsilon}}, \boldsymbol{\tau}=\frac{\partial \psi}{\partial \eta} .
$$

The PVP takes on a standard format; i.e., it encompasses only the loads doing work through the relevant (primary) degrees of freedom, as well as the concomitant stresses, namely,

$$
\int_{v}(\boldsymbol{\sigma}: \tilde{\boldsymbol{\varepsilon}}+\boldsymbol{\tau}: \tilde{\boldsymbol{\eta}}) \mathrm{d} v=\int_{v} \mathbf{b} \cdot \tilde{\mathbf{u}} \mathrm{d} v+\int_{\partial v}\left(\mathbf{t} \cdot \tilde{\mathbf{u}}+\mathbf{r} \cdot \partial_{\mathrm{n}} \tilde{\mathbf{u}}\right) \mathrm{da},
$$

which holds for any $v \subseteq V$ and for any $\tilde{\varepsilon}, \tilde{\eta}$ and $\tilde{\text { u satis- }}$ fying (3.3). The symbols $(\tilde{\sim})$ denote virtual kinematic variables. In writing the external virtual power [on the right-hand side of (3.6)], we have considered that the independent degrees of freedom are the displacements $\mathbf{u}$ over $V \cup S$ and the normal derivative of $\mathbf{u}$ over $S[4,32]$ and that therefore the set $(\mathbf{b}, \mathbf{t}, \mathbf{r})$ represents the most general set of loads that do work through, respectively, $\mathbf{u}$ in $V, \mathbf{u}$ on $S$ and $\partial_{n} \mathbf{u}$ on $S$. Using well-known procedures $[4,32],(3.6)$ gives the equilibrium equations that, in the hypothesis of regular boundary surface and the absence of inertia forces, read:

$$
\left.\begin{array}{l}
\nabla \cdot \mathbf{T}+\mathbf{b}=\mathbf{0}, \quad \mathbf{T}:=\boldsymbol{\sigma}-\nabla \cdot \boldsymbol{\tau}, \quad \text { in } v \\
\mathbf{n} \cdot \mathbf{T}-\stackrel{\mathrm{s}}{\nabla} \cdot(\boldsymbol{n} \cdot \boldsymbol{\tau})=\mathbf{t}, \quad \mathbf{n} \otimes \mathbf{n}: \boldsymbol{\tau}=\mathbf{r}, \quad \text { on } \partial v
\end{array}\right\}
$$

where $\stackrel{\mathrm{s}}{\nabla}:=\nabla_{\mathrm{T}}+\boldsymbol{K n}$ denotes the "curved-surface gradient", the sum of the tangential gradient $\nabla_{\mathrm{T}}$ and the surface curvature contribution $K \mathbf{n}$ (at a point of the surface where the unit normal vector is $\mathbf{n}$ and the mean curvature is $K=-\nabla_{\mathrm{T}} \cdot \mathbf{n}$ ). As the above is known in the literature (see, e.g., Mindlin [4], Germain [32]), we refrain from other comments on the latter equations.

For later use, we just add the remark that the nonclassical boundary conditions associated to the traction $\mathbf{t}$ in (3.7) ${ }_{3}$ can be rewritten in an equivalent form as [74]:

$$
\mathbf{n} \cdot \mathbf{T}=\mathbf{t}^{\prime}, \stackrel{\mathrm{S}}{\nabla} \cdot(\mathbf{n} \cdot \boldsymbol{\tau})+\mathbf{t}^{\prime \prime}=\mathbf{0}, \mathbf{t}^{\prime}+\mathbf{t}^{\prime \prime}=\mathbf{t}
$$

Equation (3.8), can be recognized as the tensor-valued equilibrium equation of a material surface subjected to the (symmetric) stress $\mathbf{n} \cdot \boldsymbol{\tau}$ and to the surface body force density $\mathbf{t}^{\prime \prime}$ [75]. The relations in (3.8) show that the material microstructure induces the boundary surface $\partial v$ of any subdomain $v \subseteq V$ to operate as a surface layer capable of sustaining a part, say $\mathbf{t}^{\prime \prime}$, of the traction $\mathbf{t}$, whereas the remaining part $\mathbf{t}^{\prime}$ is sustained by the bulk material through the classical Cauchy formula (3.8) [74].

The above elastic materials belong to the class of materials studied by Toupin [2], Green and Rivlin [76], Mindlin [4] and Mindlin and Eshel [5] and precisely to the class of materials addressed by the latter authors under the label “class II". For a material with free energy $\psi$ as

$$
\dot{\psi}=\frac{1}{2} \varepsilon: \mathbf{E}: \varepsilon+\frac{1}{2} \eta \vdots \mathbf{M} \vdots \eta
$$

where $\mathbf{E}$ is the usual elasticity fourth-order tensor and $\mathbf{M}$ is an analogous sixth-order tensor, the elasticity laws (3.5) read:

$$
\sigma=\mathbf{E}: \varepsilon, \quad \tau=\mathbf{M}: \eta
$$

In the case of isotropic materials, Mindlin [4] expressed $\mathbf{M}$ in terms of only five material constants, whereas $\mathbf{E}$ contains only the two Lamé constants.

\subsection{Elastically gradient materials: Aifantis model $[23,29]$}

As discussed by Forest and Aifantis [36], a particular class of second-grade materials is obtained if we assume that the relation (3.3) is satisfied even at the constitutive level and $\mathbf{M}$ is taken in the simpler form

$$
\mathbf{M}=\left\{M_{\text {kijcab }}\right\}=a^{2}\left\{E_{i j a b} \delta_{k c}\right\}
$$


where $\delta_{i j}$ is the Kronecker symbol and $a$ is a material constant (with dimension of a length). Then, (3.10) becomes

$$
\tau=a^{2} \nabla \sigma, \tau_{k i j}=a^{2} \partial_{k} \sigma_{i j}=a^{2} E_{i j a b} \partial_{k} \varepsilon_{a b},
$$

which means that the higher-order stress $\tau$ can be expressed in terms of the lower-order stress $\sigma$. Hence, the total stress $\mathbf{T}$ in (3.7) becomes

$$
\mathrm{T}=\boldsymbol{\sigma}-a^{2} \Delta \sigma=\mathrm{E}:\left(\varepsilon-a^{2} \Delta \varepsilon\right)
$$

where $\Delta=\nabla \cdot \nabla$ denotes the Laplacian operator. The latter equation relates $\mathbf{T}$ to $\varepsilon$ through a tensor-valued secondorder PDE. It characterizes a subclass of second-grade materials being elastically gradient and featured by just one material constant more than classical elasticity. These material models were advanced by Aifantis [23, 29]; see also Lazar et al. [77].

Let us remark that the above Aifantis model leads to a fourth-order displacement PDE system of the form $L \mathbf{u}=\mathbf{f}$ to solve the relevant boundary-value problem, which proves to be formally coincident with that given by the more general class of second-grade materials (3.10). Indeed, the difference just consists in the particular choice for $\mathbf{M}$ as in (3.11) with the reduction of the extra material constants to just a single one (i.e., the internal length $a$ ). The boundary conditions (including the higher-order ones) remain the same for both models, i.e., (3.7) ${ }_{3}$ and (3.7) ${ }_{4}$.

\subsection{The Aifantis elastically gradient model - an ER-based formulation}

In what precedes, the higher-order strain $\eta$ has been considered, at the constitutive level, independent of the lowerorder strain $\varepsilon$, and the Aifantis model has been derived as a particularization of the local-type material. Instead, here $\eta$ is treated as the gradient of $\varepsilon$ at the constitutive level; hence, $\varepsilon$ constitutes a nonlocality-source strain.

Then, the ER-based formulation proceeds by removing $\eta$ from the set of kinematically independent deformation modes, such that the stress power (3.2) reduces to, say, $\mathcal{W}=\mathbf{T}$ : $\dot{\varepsilon}$ (the symbol $\mathbf{T}$ is used here in place of $\sigma$ just for convenience), but including the dependence of $\psi$ on $\nabla \varepsilon$, such that the free energy (3.1) takes on the form $\psi=$ $\psi(\varepsilon, \nabla \varepsilon)$. Correspondingly, inequality (3.4) is replaced with the following one:

$$
\mathbf{T}: \dot{\varepsilon}-\dot{\psi}(\varepsilon, \nabla \boldsymbol{\varepsilon})+P \geq 0, P=\nabla \cdot(\tau: \dot{\varepsilon}) .
$$

Here, $\mathbf{T}$ is a symmetric second-order stress tensor; also, $P$ is the appropriate ER, $\tau=\left\{\tau_{\mathrm{kij}}\right\}$ is a third-order stress tensor, symmetric in the last two indices, and $\dot{\varepsilon}$ plays the role of nonlocality-source strain rate. On expanding the time derivative of $\psi$ we easily obtain from (3.14):

$$
\left(\mathbf{T}-\frac{\partial \psi}{\partial \boldsymbol{\varepsilon}}+\nabla \cdot \boldsymbol{\tau}\right): \dot{\boldsymbol{\varepsilon}}+\left(\boldsymbol{\tau}-\frac{\partial \psi}{\partial \nabla \boldsymbol{\varepsilon}}\right): \nabla \dot{\boldsymbol{\varepsilon}} \geq 0
$$

which, having to hold for arbitrary strain rate fields $\dot{\varepsilon}$, gives as necessary and sufficient conditions:

$$
\mathbf{T}=\frac{\partial \psi}{\partial \varepsilon}-\nabla \cdot\left(\frac{\partial \psi}{\nabla \partial \varepsilon}\right)
$$

with $\tau=\partial \psi / \partial \nabla \boldsymbol{\varepsilon}$. Indeed, inequality (3.14) leads directly to the constitutive equation (3.16) cast in the form of PDEs.

As for the boundary conditions, let us invoke the insulation condition in its nonhomogeneous form like in (2.21), written for the subdomain $v$, i.e.,

$$
\int_{v} P \mathrm{~d} a=\int_{\partial \nu} \mathbf{n} \cdot \tau: \dot{\varepsilon} \mathrm{d} a=\int_{\partial v} \mathbf{m}: \dot{\varepsilon} \mathrm{d} a,
$$

where $\mathbf{m}=\left\{\mathrm{m}_{i j}\right\}$ is a traction-like (symmetric) tensor. On replacing $\dot{\varepsilon}$ with $\nabla \dot{\mathbf{u}}$ (the symmetry features of $\tau$ and $\mathbf{m}$ allow this substitution), we obtain

$$
\int_{\partial v}(\mathbf{n} \cdot \tau-\mathbf{m}): \nabla \dot{\mathbf{u}} \mathrm{d} a=0 .
$$

The latter transformation amounts to shifting from the material microstructure to the continuum, the natural ambient for $\varepsilon$ and $\mathbf{u}$. On decomposing $\nabla$ into its normal and tangential parts, i.e., $\nabla=\mathbf{n} \partial_{n}+\nabla_{\mathrm{T}}$, we can obtain from (3.17):

$$
\int_{\partial \nu}(\mathbf{n} \otimes \mathbf{n}: \tau-\mathbf{n} \cdot \mathbf{m}) \cdot \partial_{n} \dot{\mathbf{u}} \mathrm{d} a-\int_{\partial \nu} \stackrel{\mathrm{S}}{\nabla} \cdot(\mathbf{n} \cdot \boldsymbol{\tau}-\mathbf{m}) \cdot \dot{\mathbf{u}} \mathrm{d} a=0 .
$$

This, with the positions

$$
\mathbf{r}:=\mathbf{n} \cdot \mathbf{m}, \mathbf{t}^{\prime \prime}:=-\stackrel{\mathrm{S}}{\nabla} \cdot \mathbf{m}
$$

becomes

$$
\int_{\partial \nu}(\mathbf{n} \otimes \mathbf{n}: \boldsymbol{\tau}-\mathbf{r}) \cdot \partial_{n} \dot{\mathbf{u}} \mathrm{d} a-\int_{\partial u}\left[\stackrel{\mathrm{S}}{\nabla} \cdot(\mathbf{n} \cdot \boldsymbol{\tau})+\boldsymbol{t}^{\prime \prime}\right] \cdot \mathbf{u} \mathrm{d} a=0,
$$

which implies

$$
\mathbf{n} \otimes \mathbf{n}: \tau=\mathbf{r}, \stackrel{\mathrm{S}}{\nabla} \cdot(\mathbf{n} \cdot \boldsymbol{\tau})+\mathbf{t}^{\prime \prime}=0 \quad \text { on } \partial v .
$$

The first one of the latter equations provides the higher-order boundary conditions (3.7), the second one represents the boundary layer equilibrium equations 
(3.8). We further remark that the PVP for the material model under consideration has the classical format:

$$
\int_{v} \mathbf{T}: \tilde{\boldsymbol{\varepsilon}} \mathrm{d} v=\int_{v} \mathbf{b} \cdot \tilde{\mathbf{u}} \mathrm{d} v+\int_{\partial v} \boldsymbol{t}^{\prime} \cdot \tilde{\mathbf{u}} \mathrm{d} a \quad \forall v \subseteq V,
$$

where we have written $\mathbf{t}^{\prime}$ for the Cauchy surface traction just for convenience. Thus, the equilibrium equations read

$$
\nabla \cdot \mathbf{T}+\mathbf{b}=0 \text { in } v, \mathbf{n} \cdot \mathbf{T}=\mathbf{t}^{\prime} \text { on } \partial v,
$$

which coincide with (3.7) and (3.8), respectively.

In concluding this subsection, we can realize that the (weak nonlocal) Clausius-Duhem inequality (3.14) and the insulation condition (3.17), together with the PVP in its classical format (3.23), lead us to the complete set of field and boundary equations governing the considered elastically gradient (Aifantis) material model, with the constitutive equations being cast as PDEs accompanied by the pertinent boundary conditions.

\section{Second-grade elastic-plastic models}

In this section, materials like those of Section 3 are addressed, but in the hypothesis of plastically gradient behavior. An isotropic material is first considered (which is also addressed by the PVP-approach in Subsection 4.2 for comparison), then a crystal plasticity model is presented (in Subsection 4.3).

\subsection{Plastically gradient material (ER approach)}

Let the nonsimple material treated in Subsection 3.1 be reconsidered, but with the assumption that the material is plastically gradient. The stress power is still given by (3.2). The additive strain decomposition holds, i.e., $\varepsilon=\varepsilon^{\mathrm{e}}+\varepsilon^{\mathrm{p}}$, $\eta=\eta^{\mathrm{e}}+\eta^{\mathrm{p}}$ with $\eta^{\mathrm{p}}=\nabla \boldsymbol{\varepsilon}^{\mathrm{p}}$ holding at the constitutive level. In view of the application of the ER approach let the free energy (2.6) be expressed as

$$
\psi=\psi_{e}\left(\boldsymbol{\varepsilon}-\boldsymbol{\varepsilon}^{\mathrm{p}}, \boldsymbol{\eta}-\nabla \boldsymbol{\varepsilon}^{\mathrm{p}}\right)+\psi_{h}(\kappa)
$$

where $\boldsymbol{\varepsilon}^{p}$ is a specification of the pseudo-internal variable $\gamma$, and $\kappa$ denotes a scalar (true) internal variable with the meaning of effective plastic strain (the variables $\chi$ and $\kappa$ are here identically vanishing). As far as the evolution law of the material is unspecified, the actual relation of $\kappa$ to $\varepsilon^{\mathrm{p}}$ is unknown, it is thus treated as independent of $\varepsilon^{p}$ in the search for the thermodynamic restrictions on the constitutive equations; in this way, the latter restrictions do hold for whatsoever choice of the evolution/diffusion laws. Then, the (weak nonlocal) Clausius-Duhem inequality (2.10) can be written, in isothermal conditions, as [see (3.4) for comparison]:

$$
D=\sigma: \dot{\varepsilon}+\tau \quad \vdots \dot{\eta}-\dot{\psi}+P \geq 0,
$$

where $P$ is the ER. Since $\varepsilon^{p}$ is the only nonlocality-source strain, we can express $P$ as

$$
P=\nabla \cdot\left(\mathbf{S}: \dot{\varepsilon}^{\mathrm{p}}\right)
$$

where $\mathbf{S}$ is a third-order stress tensor, symmetric in the two last indices. On expanding the time derivative of $\psi$, we obtain from (4.2):

$$
\begin{aligned}
D & =\left(\boldsymbol{\sigma}-\frac{\partial \psi_{\mathrm{e}}}{\partial \boldsymbol{\varepsilon}}\right): \dot{\boldsymbol{\varepsilon}}+\left(\boldsymbol{\tau}-\frac{\partial \psi_{\mathrm{e}}}{\partial \boldsymbol{\eta}}\right): \dot{\boldsymbol{\eta}}+\left(\nabla \cdot \mathbf{S}-\frac{\partial \psi_{\mathrm{e}}}{\partial \boldsymbol{\varepsilon}^{\mathrm{P}}}\right): \dot{\boldsymbol{\varepsilon}}^{\mathrm{P}} \\
& +\left(\mathbf{S}-\frac{\partial \psi_{\mathrm{e}}}{\partial \nabla \boldsymbol{\varepsilon}^{\mathrm{P}}}\right): \nabla \dot{\boldsymbol{\varepsilon}}^{\mathrm{P}}-\frac{\partial \psi_{\mathrm{h}}}{\partial \kappa} \dot{\kappa} \geq 0 .
\end{aligned}
$$

The latter inequality holds for any deformation mechanism and evolution law. Then, considering purely elastic deformation mechanisms, (4.4) loses all the plastic contributions and gives

$$
\boldsymbol{\sigma}=\frac{\partial \psi_{\mathrm{e}}}{\partial \boldsymbol{\varepsilon}}, \boldsymbol{\tau}=\frac{\partial \psi_{\mathrm{e}}}{\partial \eta} .
$$

These relations are the elasticity laws, which are assumed to hold also in the general case of elastic-plastic deformation mechanisms. Hence, with the positions:

$$
\left.\begin{array}{l}
\mathbf{T}:=\boldsymbol{\sigma}-\nabla \cdot \boldsymbol{\tau}, R:=\frac{\partial \psi_{\mathrm{h}}}{\partial \kappa} \\
\boldsymbol{\sigma}^{\mathrm{P}}:=-\frac{\partial \psi_{\mathrm{e}}}{\partial \nabla \boldsymbol{\varepsilon}^{\mathrm{P}}}=\boldsymbol{\sigma}, \boldsymbol{\tau}^{\mathrm{P}}:=-\frac{\partial \psi_{\mathrm{e}}}{\partial \nabla \boldsymbol{\varepsilon}^{\mathrm{P}}}=\boldsymbol{\tau} \\
\mathbf{T}^{\mathrm{P}}=\boldsymbol{\sigma}^{\mathrm{P}}-\nabla \cdot \boldsymbol{\tau}^{\mathrm{P}}=\mathbf{T}, \\
\mathbf{T}^{\mathrm{D}}:=\mathbf{T}^{\mathrm{P}}+\nabla \cdot \mathbf{S}^{\mathrm{D}}, \mathbf{S}^{\mathrm{D}}:=\mathbf{S}+\boldsymbol{\tau}^{\mathrm{P}}
\end{array}\right\}
$$

inequality (4.4) reduces to

$$
D=\mathbf{T}^{\mathrm{D}}: \dot{\boldsymbol{\varepsilon}}^{\mathrm{p}}+\mathbf{S}^{\mathrm{D}}: \nabla \dot{\boldsymbol{\varepsilon}}^{\mathrm{p}}-R \dot{\kappa} \geq 0 .
$$

As shown by (4.6), $\mathbf{T}^{\mathrm{p}}$ denotes the stress $\mathbf{T}$ built with the dissipative stresses $\boldsymbol{\sigma}^{\mathrm{p}}$ and $\boldsymbol{\tau}^{\mathrm{p}}$. The stress $\mathbf{S}^{\mathrm{D}}$ is associated to the higher-order dissipation modes, hence to the moving geometrically necessary dislocations (GNDs) macroscopically represented by $\nabla \dot{\boldsymbol{\varepsilon}}^{\mathrm{p}}[78]$, whereas the stress $\mathbf{T}^{\mathrm{D}}:=\mathbf{T}+\nabla \cdot \mathbf{S}^{\mathrm{D}}$ is associated to the lower-order dissipation modes, hence to the moving statistically stored dislocations (SSDs) macroscopically represented by $\dot{\boldsymbol{\varepsilon}}^{\mathrm{P}}$. Also, the 
isotropic hardening stress, $R$, is associated to the inherent irreversibly stored energy, hence to the cumulating SSDs macroscopically represented by $\dot{\kappa}$. The stresses $\mathbf{T}$ and $\tau$ are required to satisfy the equilibrium equations (3.7), as it descends from the fact that the PVP for the structural system under consideration is the same as in Subsection 3.1.

Equations (4.5) and (4.6) provide the state equations for all the stress variables, except for $\mathbf{S}^{\mathrm{D}}$, which will be determined only by the evolution laws. In the case of rateindependent and associative plasticity, these laws can be expressed starting, for instance, with a yield function, say

$$
f=\sqrt{\left\|\mathbf{T}^{\mathrm{p}}+\nabla \cdot \mathbf{S}^{\mathrm{D}}\right\|^{2}+\frac{1}{l^{2}}\left\|\mathbf{S}^{\mathrm{D}}\right\|^{2}-R-\sigma_{0}}
$$

where $l$ is an internal length parameter and $\sigma_{0}$ is the yield strength. Then, with the normality rule and a standard notation we can write:

$$
\dot{\boldsymbol{\varepsilon}}^{\mathrm{p}}=\dot{\lambda} \frac{\mathbf{T}^{\mathrm{p}}+\nabla \cdot \mathbf{S}^{\mathrm{D}}}{R+\sigma_{0}}, \nabla \dot{\boldsymbol{\varepsilon}}^{\mathrm{p}}=\frac{\dot{\lambda}}{l^{2}} \frac{\mathbf{S}^{\mathrm{D}}}{R+\sigma_{0}}, \dot{\kappa}=\dot{\lambda}
$$

which holds for $f=0$; otherwise, it is $f<0$, then $\dot{\lambda}=0$ and $\mathbf{S}^{\mathrm{D}}=\mathbf{0}$ (no moving GNDs in the absence of any plastic deformation mechanism). In the region of $V$ where $\dot{\lambda}>0$, we easily obtain from (4.9):

$$
\dot{\kappa}=\varphi:=\sqrt{\left\|\dot{\varepsilon}^{\mathrm{p}}\right\|^{2}+l^{2}\left\|\nabla \dot{\boldsymbol{\varepsilon}}^{\mathrm{p}}\right\|^{2}}
$$

which specifies the dependence of $\dot{\kappa}$ on $\dot{\boldsymbol{\varepsilon}}^{\mathrm{P}}$. Additionally, solving (4.9), for $\mathbf{S}^{\mathrm{D}}$ and then substituting into (4.9) gives, by (4.9) ${ }_{3}$ and (4.10),

$$
\mathbf{T}^{\mathrm{p}}=\left(\sigma_{0}+R\right)\left[\frac{\dot{\varepsilon}^{\mathrm{p}}}{\varphi}-l^{2} \nabla \cdot\left(\frac{\nabla \dot{\boldsymbol{\varepsilon}}^{\mathrm{p}}}{\varphi}\right)\right]-l^{2} \frac{\nabla R \cdot \nabla \dot{\boldsymbol{\varepsilon}}^{\mathrm{p}}}{\varphi},
$$

where $R$ is a function of $k=\int_{0}^{t} \varphi d t$. Eq. (4.11) is the evolution-diffusion equation of the material; it is similar to one advanced by Polizzotto [58] for strengthening materials, whereas in the case of perfectly plastic material $(R=0)$, (4.11) coincides with analogous equations given by Gurtin [44, 45] and Anand et al. [47].

At a fixed time, in which the state of the material is known (hence $\mathbf{T}^{\mathrm{p}}$ and $R$ are some fixed fields), (4.11) constitutes a PDE system that can be solved in terms of the strain rate field $\dot{\boldsymbol{\varepsilon}}^{\mathrm{P}}$. The higher-order boundary conditions needed for this purpose can be readily obtained by invoking the insulation condition, for instance in its homogeneous form, i.e., by (4.3):

$$
\int_{V} P \mathrm{~d} v=\int_{S} \mathbf{n} \cdot \mathbf{S}: \dot{\varepsilon}^{\mathrm{p}} \mathrm{d} a=0
$$

As this is an identity to be satisfied for any deformation mechanism, assuming that $\dot{\boldsymbol{\varepsilon}}^{\mathrm{P}} \neq \mathbf{0}$ within some region $V_{p} \subset V$, we can write as a consequence of (4.12) and using the expression of $\mathbf{S}$ taken from $(4.6)_{4}$ with $\mathbf{S}^{\mathrm{D}}$ derived from $(4.9)_{2}$ :

$$
\left.\begin{array}{l}
\dot{\boldsymbol{\varepsilon}}^{\mathrm{p}}=\mathbf{0} \text { on } S_{\mathrm{ph}}, \mathbf{n} \cdot \mathbf{S}=l^{2}\left(\mathrm{R}+\sigma_{0}\right) \frac{\partial_{\mathrm{n}} \dot{\varepsilon}^{\mathrm{p}}}{\varphi}-\mathbf{n} \cdot \boldsymbol{\tau}^{\mathrm{p}}=\mathbf{0} \text { on } S_{\mathrm{pf}} \\
\dot{\varepsilon}^{\mathrm{p}}=l^{2}\left(\mathrm{R}+\sigma_{0}\right) \frac{\partial_{\mathrm{n}} \dot{\varepsilon}^{\mathrm{p}}}{\varphi}-\mathbf{n} \cdot \boldsymbol{\tau}^{\mathrm{p}}=\mathbf{0} \text { on } S_{\mathrm{p}(\mathrm{int})}
\end{array}\right\}
$$

where $S_{\mathrm{ph}} \cup S_{\mathrm{pf}} \cup S_{\mathrm{p}(\mathrm{int})}=S_{p}:=\partial V_{p}$, and $S_{\mathrm{p}(\mathrm{int})}$ is the moving elastic-plastic boundary surface. Eq. (4.13) generalizes to second-grade elastic-plastic materials analogous conditions given by Polizzotto [58] for first-grade materials (for which $\left.\tau^{\mathrm{p}}=\mathbf{0}, \varphi=\left\|\dot{\boldsymbol{\varepsilon}}^{\mathrm{P}}\right\|\right)$. The above boundary conditions are rather cumbersome mainly due to the moving elastic/ plastic boundary, for which a strategy named "implicit gradient plasticity" may be a remedy [79].

Dropping the isotropic hardening $(R=0)$, the above elastic-plastic material model can be compared to the one proposed by Fleck and Hutchinson [28] for secondgrade Toupin-Mindlin elastoplasticity, where the higherorder plastic strain rate, $\dot{\eta}^{\mathrm{P}}$, is not guaranteed to coincide with the gradient of $\dot{\boldsymbol{\varepsilon}}^{\mathrm{P}}$, whereas in the present model it is. Additionally, for $\mathbf{S}^{\mathrm{D}}=0$, but $R \neq 0$, the proposed model proves to be similar to the "strain gradient model" by Forest and Sievert [8].

\subsection{Plastically gradient material - PVP approach}

It seems appropriate here to address the same material model treated in the previous subsection by means of the PVP approach, which will give us the opportunity to complete the comparison of Subsection 2.4. For this purpose, let us note that the pseudo-internal variables $\gamma$ and $\nabla \gamma$ are here replaced by the analogous variables $\varepsilon^{p}$ and $\eta^{p}$, respectively, whereas the variables $\chi$ and $\kappa$, are lacking. The PVP approach starts - as already specified in Subsection 2.4 with the following steps:

1. It treats the pseudo-internal variables $\varepsilon^{\mathrm{p}}$ and $\boldsymbol{\eta}^{\mathrm{p}}$ as independent of each other at the constitutive level, but related by the compatibility relation $\eta^{\mathrm{p}}=\nabla \boldsymbol{\varepsilon}^{\mathrm{p}}$ at the global structural level, with $\varepsilon^{\mathrm{p}}$ having the role of extra degrees of freedom.

2. Correspondingly, it also considers an enlarged stress power of the form

$$
\mathcal{W}_{\text {enl }}=\mathcal{W}+\mathcal{W}^{+}
$$


where $\mathcal{W}$ is given by (3.2), whereas $\mathcal{W}^{+}$is the extra stress power expressed as

$$
\mathcal{W}^{+}=\pi: \dot{\varepsilon}^{\mathrm{P}}+\mathbf{S}: \dot{\eta}^{\mathrm{P}}
$$

with $\pi$ and $\mathbf{S}$ being some suitable microstress tensors, respectively, of second and third order.

Since $\dot{\boldsymbol{\varepsilon}}^{\mathrm{P}}$ and $\dot{\eta}^{\mathrm{P}}$ are mutually related by a compatibility condition, necessarily $\pi$ and $\mathbf{S}$ must satisfy some related equilibrium equations. The PVP approach accomplishes the task of writing out the latter equations by the extended PVP. In the present case, this PVP reads as

$$
\begin{gathered}
\int_{v}\left(\boldsymbol{\sigma}: \tilde{\boldsymbol{\varepsilon}}+\boldsymbol{\tau}: \tilde{\boldsymbol{\eta}}+\boldsymbol{\pi}: \tilde{\boldsymbol{\varepsilon}}^{\mathrm{p}}+\mathbf{S}: \tilde{\boldsymbol{\eta}}^{\mathrm{p}}\right) \mathrm{d} v=\int_{v} \mathbf{b} \cdot \tilde{\mathbf{u}} \mathrm{d} v \\
+\int_{\partial v}\left(\mathbf{t} \cdot \tilde{\mathbf{u}}+\mathbf{r} \cdot \partial_{n} \tilde{\mathbf{u}}+\mathbf{m}: \tilde{\boldsymbol{\varepsilon}}^{\mathrm{p}}\right) \mathrm{d} a
\end{gathered}
$$

where $\mathbf{m}$ is a symmetric second-order tensor denoting moment traction on $\partial v$, whereas $\tilde{\boldsymbol{\varepsilon}}, \tilde{\boldsymbol{\eta}}, \tilde{\boldsymbol{\varepsilon}}^{\mathrm{P}}, \tilde{\boldsymbol{\eta}}^{\mathrm{P}}$ and $\tilde{\mathbf{u}}$ are arbitrary virtual kinematic variables that satisfy the compatibility equations

$$
\tilde{\boldsymbol{\varepsilon}}=\operatorname{sym} \nabla \tilde{\mathbf{u}}, \tilde{\boldsymbol{\eta}}=\nabla \tilde{\boldsymbol{\varepsilon}}, \tilde{\boldsymbol{\eta}}^{\mathrm{p}}=\nabla \tilde{\boldsymbol{\varepsilon}}^{\mathrm{p}} .
$$

By a well-known procedure similar to the one invoked for (3.6), we can easily obtain the equilibrium equations that include (3.7), as well as the microstress equilibrium equations, i.e.,

$$
\boldsymbol{\pi}=\nabla \cdot \mathbf{S}, \mathbf{n} \cdot \mathbf{S}=\mathbf{m}
$$

which are formally identical to (2.14).

Furthermore, within the PVP approach in consideration, the Clausius-Duhem inequality is enforced in a localtype form (i.e., in isothermal conditions)

$$
D=\mathcal{W}_{\text {enl }}-\dot{\psi} \geq 0
$$

where $\psi$ has the form:

$$
\psi=\psi_{\mathrm{e}}\left(\boldsymbol{\varepsilon}-\boldsymbol{\varepsilon}^{\mathrm{p}}, \boldsymbol{\eta}-\boldsymbol{\eta}^{\mathrm{p}}\right)+\psi_{\mathrm{h}}(\kappa) .
$$

This $\psi$ is similar to (4.1), but here $\boldsymbol{\eta}^{\mathrm{p}}$ is considered independent of $\boldsymbol{\varepsilon}^{\mathrm{p}}$ at the constitutive level.

On expanding the time derivative of $\psi$, Eq. (4.19) gives

$$
\begin{aligned}
D & =\left(\boldsymbol{\sigma}-\frac{\partial \psi_{\mathrm{e}}}{\partial \boldsymbol{\varepsilon}}\right): \dot{\boldsymbol{\varepsilon}}+\left(\boldsymbol{\tau}-\frac{\partial \psi_{\mathrm{e}}}{\partial \boldsymbol{\eta}}\right): \dot{\boldsymbol{\eta}}+\left(\pi-\frac{\partial \psi_{\mathrm{e}}}{\partial \boldsymbol{\varepsilon}^{\mathrm{p}}}\right): \dot{\boldsymbol{\varepsilon}}^{\mathrm{p}} \\
& +\left(\mathbf{S}-\frac{\partial \psi_{\mathrm{e}}}{\partial \boldsymbol{\eta}^{\mathrm{p}}}\right): \dot{\boldsymbol{\eta}}^{\mathrm{p}}-\frac{\partial \psi^{\mathrm{h}}}{\partial \kappa} \dot{\kappa} \geq 0
\end{aligned}
$$

which parallels inequality (4.4). Like the latter, inequality (4.21) gives the elasticity laws (4.5); thus, using the notation (4.6) $)_{2,3,4}$ it simplifies as

$$
D=(\boldsymbol{\pi}+\boldsymbol{\sigma}): \dot{\boldsymbol{\varepsilon}}^{\mathrm{p}}+(\mathbf{S}+\boldsymbol{\tau}): \dot{\boldsymbol{\eta}}^{\mathrm{p}}-R \dot{\kappa} \geq 0 .
$$

Next, making use of the microstress equilibrium equation (4.18) (by which the redundant stress $\pi$ can be eliminated) and using the notation (4.6) $)_{5,6,7}$ inequality (4.22) can be transformed into:

$$
D=\mathbf{T}^{\mathrm{D}}: \dot{\boldsymbol{\varepsilon}}^{\mathrm{p}}+\mathbf{S}^{\mathrm{D}}: \dot{\boldsymbol{\eta}}^{\mathrm{p}}-R \dot{\kappa} \geq 0 .
$$

This is like (4.7) in all, except for the second dissipation addend here expressed in terms of the higherorder strain rate $\dot{\eta}^{\mathrm{P}}$ instead of the strain rate gradient counterpart $\nabla \dot{\boldsymbol{\varepsilon}}^{\mathrm{P}}$ (a difference that may have some consequences within the numerical treatment of the plasticity laws under consideration). From this point on, the two approaches proceed in parallel in the task of formulating the evolution/diffusion laws of the material.

In conclusion, we can state that, on one hand, the ER approach leads to the state equations and to the pertinent dissipation inequality in a more direct way, for it does require the PVP just in its standard format like (3.6) or even does not require it at all whenever (as it is often the case) the inherent standard equilibrium equations are already known. This is made possible by the involvement of the ER $P$, whose constitutive form contains the basic piece of information provided by the mentioned microstress equilibrium equations. Indeed, with the ER approach, the basic content of the microstress equilibrium equations is, once and for all, incorporated into the representation formula of $P$ in (2.15), or (4.3), whereby only the independent variables, i.e., the nonlocalitysource strain rate $\left(\dot{\varepsilon}^{\mathrm{P}}\right)$ and the related higher-order microstress $(\mathbf{S})$, are retained.

On the other hand, the PVP approach introduces a richer set of microstresses $(\pi, \mathbf{S})$ and eliminates the redundant one $(\pi)$ by the microstress equilibrium equations (4.18), the latter being obtained by means of the extended PVP. Therefore, we can state that the PVP approach leads to (substantially) the same results as the ER approach, but through a less simple procedure whereby a larger number of additional state variables is required and the (often already known) standard equilibrium equations are also determined.

The latter features of the PVP approach are pointed out more clearly if one considers that, as explained by Polizzotto [58], the (extended) PVP (4.16) can be split into two distinct PVPs, namely, the standard PVP (3.6) and the inner PVP involving only the pertinent microstresses and microtractions together with the related kinematic variables, i.e.:

$$
\int_{v}\left(\pi: \tilde{\varepsilon}^{\mathrm{p}}+\mathbf{S}: \tilde{\boldsymbol{\eta}}^{\mathrm{p}}\right) \mathrm{d} v=\int_{\partial v} \mathbf{m}: \tilde{\boldsymbol{\varepsilon}}^{\mathrm{p}} \mathrm{d} a .
$$


This holds for arbitrary $v \subseteq V$ and for arbitrary virtual plastic strain fields $\tilde{\boldsymbol{\varepsilon}}^{\mathrm{P}}$ under the constraint $\tilde{\boldsymbol{\eta}}^{\mathrm{P}}=\nabla \tilde{\boldsymbol{\varepsilon}}^{\mathrm{P}}$; it thus implies (4.18). Using (4.24) in place of (4.16) makes it possible for us to extract from the inner PVP the same piece of information, i.e., (4.18), extracted from the extended PVP and needed for the constitutive equation formulation. In this way, the standard PVP would just remain available for use whenever the standard equilibrium equations have to be determined; nevertheless, there also remains the fact that the inner PVP (2.24) carries in the redundant state variable $\pi$.

\subsection{Plastically gradient single crystals}

The material model treated in Subsection 4.1 can be readily extended to single crystal materials with the usual assumption that plastic deformation occurs in the form of slips along prefixed directions on a set of prefixed slip planes. Denoting by $\boldsymbol{v}^{\alpha}$ the unit normal vector to the $\alpha$-th slip plane and by $\mathbf{s}^{\alpha}$ the unit vector in the slip direction, we can write $[43,80,81]$ :

$$
\boldsymbol{\varepsilon}^{\mathrm{p}}=\sum_{\alpha} \gamma^{\alpha} \mathbf{M}^{\alpha}, \nabla \boldsymbol{\varepsilon}^{\mathrm{p}}=\sum_{\alpha} \nabla \gamma^{\alpha} \mathbf{M}^{\alpha}
$$

where

$$
\mathbf{M}^{\alpha}:=\operatorname{sym}\left(\mathbf{s}^{\alpha} \otimes \boldsymbol{v}^{\alpha}\right),(\alpha=1,2, \ldots, N) .
$$

In view of the application of the ER approach, let us express the free energy (2.6) as

$$
\psi=\psi_{e}\left(\varepsilon-\varepsilon^{p}, \eta-\eta^{p}\right)+\psi_{\mathrm{h}}\left(\gamma^{\alpha}, \nabla \gamma^{\alpha}\right),
$$

where $\psi_{\mathrm{h}}$ includes all the slip variables $\gamma^{\alpha}$ and their gradients, like in Nicola et al. [82]. The $\gamma^{\alpha}$ 's constitute a set of scalar pseudo-internal variables.

If necessary, $\nabla \gamma^{\alpha}$ may be replaced with the tangential gradient on the $\alpha$-th plane, i.e., the quantity

$$
\nabla_{\mathrm{T}} \gamma^{\alpha}=\nabla \gamma^{\alpha}-\boldsymbol{v}^{\alpha} \gamma_{, v^{\alpha}}^{\alpha}, \quad(\alpha=1,2, \ldots, N),
$$

which is known [28] to represent the GNDs, or consider $\nabla_{\mathrm{T}} \gamma^{\alpha}$ and $\gamma_{,,^{\alpha}}^{\alpha}$ as distinct arguments of $\psi_{\mathrm{h}}$, but for simplicity here we disregard such details. Also, let the ER $P$ be cast in the form

$$
P=\sum_{\alpha} \nabla \cdot\left(\mathbf{q}^{\alpha} \dot{\gamma}^{\alpha}\right)
$$

where the $\mathbf{q}^{\alpha}$ 's denote some vector-valued (resolved) longrange stresses. Then, the Clausius-Duhem inequality, still of the form (4.2), but $\psi$ given by (4.27), leads to the elasticity laws (4.5) and to a dissipation inequality as

$$
\begin{aligned}
D & =\sum_{\alpha}\left[\left(\boldsymbol{\sigma}^{\mathrm{p}}: \mathbf{M}^{\alpha}+\nabla \cdot \mathbf{q}^{\alpha}-\frac{\partial \psi_{\mathrm{h}}}{\partial \gamma^{\alpha}}\right) \dot{\gamma}^{\alpha}\right. \\
& \left.+\left(\boldsymbol{\tau}^{\mathrm{p}}: \mathbf{M}^{\alpha}+\mathbf{q}^{\alpha}-\frac{\partial \psi_{\mathrm{h}}}{\partial \nabla \gamma^{\alpha}}\right) \cdot \nabla \dot{\gamma}^{\alpha}\right] \geq 0
\end{aligned}
$$

where $\sigma^{\mathrm{p}}$ and $\tau^{\mathrm{p}}$ coincide with the analogous definitions in (4.6). In similarity to Gurtin et al. [83], let us posit:

$$
\mathbf{q}_{\mathrm{D}}^{\alpha}:=\boldsymbol{\tau}^{\mathrm{P}}: \mathbf{M}^{\alpha}+\mathbf{q}^{\alpha}-\frac{\partial \psi_{\mathrm{h}}}{\partial \nabla \gamma^{\alpha}}, \quad(\alpha=1,2, \ldots, N)
$$

moreover

$$
\left.\begin{array}{l}
\sigma^{\alpha}:=\sigma: \mathbf{M}^{\alpha}, \quad \tau^{\alpha}:=\tau: \mathbf{M}^{\alpha} \\
T^{\alpha}:=\boldsymbol{\sigma}^{\alpha}-\nabla \cdot \tau^{\alpha}=\mathbf{T}: \mathbf{M}^{\alpha} \\
X^{\alpha}:=\frac{\partial \psi_{\mathrm{h}}}{\partial \gamma^{\alpha}}-\nabla \cdot\left(\frac{\partial \psi_{\mathrm{h}}}{\partial \nabla \gamma^{\alpha}}\right)
\end{array}\right\} \quad(\alpha=1,2, \ldots, N)
$$

where $\mathbf{T}$ is given by (4.6). Then, inequality (4.30) becomes

$$
D=\sum_{\alpha}\left[\left(T^{\alpha}-X^{\alpha}+\nabla \cdot \mathbf{q}_{\mathrm{D}}^{\alpha}\right) \dot{\gamma}^{\alpha}+\mathbf{q}_{\mathrm{D}}^{\alpha} \cdot \nabla \dot{\gamma}^{\alpha}\right] \geq 0
$$

which gives $D$ as the sum of contributions coming from every slip plane, similar to that in Gurtin et al. [83]. The symbol $X^{\alpha}$ denotes the $\alpha$-th resolved back stress in (4.32) ${ }_{5}$. The latter equation is a second-order PDE expressing $X^{\alpha}$ in terms of $\gamma^{\alpha}$.

The associated boundary conditions can be obtained by the insulation condition, which in its homogeneous form reads, by (4.29),

$$
\int_{S} \sum_{\alpha} \mathbf{n} \cdot \mathbf{q}^{\alpha} \dot{\gamma}^{\alpha} \mathrm{d} \alpha=0
$$

This suggests us to write the boundary conditions as

$$
\dot{\gamma}^{\alpha}=0 \text { on } \mathrm{S}_{\mathrm{h}}, \mathbf{n} \cdot \mathbf{q}^{\alpha}=0 \text { on } \mathrm{S}_{\mathrm{f}},(\alpha=1,2, \cdots, N) .
$$

However, Gurtin and Needleman [84] found that the condition of null slip rates on the boundary surface of the crystal may be too restrictive for the solution existence. Then, we relax it by assuming that there exists a thirdorder tensor $\mathbf{Q}=\left\{Q_{k i j}\right\}$ such that

$$
\mathbf{q}^{\alpha}=\mathbf{Q}: \mathbf{M}^{\alpha},(\alpha=1,2, \ldots, N) .
$$

Equation (4.34) can thus be replaced with

$$
\int_{S} \mathbf{n} \cdot \mathbf{Q}: \dot{\varepsilon}^{\mathbf{P}} \mathrm{d} a=0,
$$


and therefore the boundary conditions read

$$
\dot{\boldsymbol{\varepsilon}}^{\mathrm{p}}=\mathbf{0} \text { on } S_{\mathrm{h}}, \mathbf{n} \cdot \mathbf{Q}=\mathbf{0} \text { on } S_{\mathrm{f}} .
$$

Note that the Neumann-type boundary conditions $(4.35)_{2}$ and (4.38) ${ }_{2}$ are, by (4.36), equivalent to each other.

\section{Material models with microstructure}

Materials with microstructure were introduced independently by Toupin [2], Eringen and Suhubi [9] and Mindlin [4]. Germain [32] envisioned an extended form of the PVP to address the richer mechanics of such materials, often referred to as "micromorphic" materials after Eringen and Suhubi [9]. Aifantis [15] treated materials with microstructure within a general thermodynamic framework in which he advanced the pioneering concept of "extra internal energy" (similar to our ER).

A typical micromorphic material model is conceived as a macroscopic continuum (or macrostructure) made up of particles to each of which a microscopic continuum of small extent is attached, distinguished with names as “cell” after Mindlin [4], or "microstructure" after Germain [32]. Both the macro- and the microstructure can deform independently from each other; hence, distinct variables are required to describe their strain and stress states. However, whereas the macrostructure is required to deform in a compatible manner through the displacement field $\mathbf{u}$, no such requirement is demanded of the microstructure. Forest and Sievert [8] and Forest [35] provided a complete PVP-based theory (referred to as "micromorphic approach") for these materials. Here we want to show that the ER-based theory can be applied with some advantages. For this purpose, we shall address a few elastic and elastic-plastic models previously studied by Forest and Sievert [8] and Forest [35].

\subsection{Local-type elastic material with local-type elastic microstructure}

Let the deformation of the macroscopic continuum be described by the distortion tensor $\mathbf{H}=\nabla \mathbf{u}$. Also, let the microstructure undergo an independent deformation described by a strain-like tensor $\chi$, which in practice can be a tensor of any order (even a vector, or a scalar), but is here formally treated as a nonsymmetric second-order tensor. $\chi$ has the role of (secondary) degrees of freedom $(\xi \equiv \chi)$ related to the (absolute) motion of the microstructure. This $\chi$ can be cast in the form

$$
\chi=\beta+\chi^{\mathrm{C}}
$$

where $\beta$ is a measure of the relative deformation between the microstructure and the continuum, whereas $\chi^{\mathrm{C}}$ denotes the convected part of $\chi$, i.e., the value of $\chi$ when $\beta=0$, and proves to be some function of $\mathbf{H}$, say $\chi^{\mathrm{C}}=\chi^{\mathrm{C}}(\mathbf{H})$. The tensor $\boldsymbol{\kappa}$, describes the intrinsic deformation of the microstructure; it may be required to coincide with the gradient $\nabla \chi$. Often in the literature $[8,35,36,85,86]$ the variables related to the microstructure are rendered recognizable by attaching to them a special mark; here the distinction between such variables and those related to the continuum is left to the evidence of the context.

Although elastic micromorphic materials have been studied in the literature $[9,35,36]$, here we address them in order to get the occasion of clarifying some fundamental aspects of the ER-based theory (applied hereafter). For this purpose, let us remark that such a material can be cast within our framework by means of a free energy function as

$$
\psi=\psi_{1}(\varepsilon)+\psi_{2}(\beta, \kappa)
$$

which characterizes the micromorphic material in the (assumed) isothermal conditions. The set $(\varepsilon, \beta, \kappa)$, with $\varepsilon:=$ symH and $\boldsymbol{\kappa}$ independent of $\chi$, can be considered the set of all kinematically independent deformation modes of the material. Therefore, the stress power can be written as

$$
\mathcal{W}=\sigma: \dot{\varepsilon}+\mathbf{s}: \dot{\beta}+\mu: \dot{\kappa}
$$

where $\boldsymbol{\sigma}=\left\{\sigma_{i j}\right\}, \mathbf{s}=\left\{s_{i j}\right\}, \boldsymbol{\mu}=\left\{\mu_{k i j}\right\}$ denote stress tensors that are work-conjugate of $\dot{\boldsymbol{\varepsilon}}, \dot{\boldsymbol{\beta}}$ and $\dot{\boldsymbol{\kappa}}$, respectively, with $\sigma$ being symmetric. As shown by Germain [32] and Forest [35], the latter stress power is invariant under change of observer. In fact, under a transformation of the type (2.4), we have $\dot{\mathbf{H}} \rightarrow \dot{\mathbf{W}}, \dot{\chi} \rightarrow \dot{\chi}^{\mathrm{C}}(\mathbf{W})$, hence

$$
\dot{\boldsymbol{\varepsilon}} \rightarrow \mathbf{0}, \dot{\boldsymbol{\beta}} \rightarrow \mathbf{0}, \dot{\boldsymbol{\kappa}}=\nabla \dot{\chi} \rightarrow \nabla \dot{\chi}^{\mathrm{C}}=\frac{\partial \dot{\chi}^{\mathrm{C}}}{\partial \mathbf{W}}:(\nabla \dot{\mathbf{W}})^{T}=\mathbf{0} .
$$

In the absence of any nonlocality-source strain, no ER is needed and the Clausius-Duhem inequality (2.10) takes on the local-type form:

$$
\sigma: \dot{\varepsilon}+\mathbf{S}: \dot{\beta}+\mu: \kappa-\dot{\psi}(\varepsilon, \beta, \kappa) \geq 0 .
$$

Inequality (5.5), following a standard procedure, gives the state equations:

$$
\boldsymbol{\sigma}=\frac{\partial \psi_{1}}{\partial \boldsymbol{\varepsilon}}, \mathbf{s}=\frac{\partial \psi_{2}}{\partial \boldsymbol{\beta}}, \boldsymbol{\mu}=\frac{\partial \psi_{2}}{\partial \boldsymbol{\kappa}}
$$


which define the elasticity laws for the macro- and microstructure.

Following Germain [32] and Forest and Aifantis [36], the PVP can be rewritten as

$$
\int_{v}(\boldsymbol{\sigma}: \tilde{\boldsymbol{\varepsilon}}+\mathbf{S}: \tilde{\boldsymbol{\beta}}+\boldsymbol{\mu}: \tilde{\boldsymbol{\kappa}}) \mathrm{d} v=\int_{v} \boldsymbol{b} \cdot \tilde{\mathbf{u}} \mathrm{d} v+\int_{v}(\mathbf{t} \cdot \tilde{\mathbf{u}}+\mathbf{m}: \tilde{\chi}) \mathrm{d} a
$$

which holds under the constraints

$$
\tilde{\mathbf{H}}=\nabla \tilde{\mathbf{u}}, \quad \tilde{\boldsymbol{\varepsilon}}=\operatorname{sym} \tilde{\mathbf{H}}, \quad \tilde{\beta}=\tilde{\chi}-\chi^{\mathrm{C}}(\tilde{\mathbf{H}}), \quad \tilde{\boldsymbol{\kappa}}=\nabla \tilde{\chi} .
$$

Due to the symmetry of $\sigma, \tilde{\varepsilon}$ can be replaced with $\nabla \tilde{\mathbf{u}}$. Then, applying the divergence theorem and with the positions:

$$
\tau:=\boldsymbol{\sigma}-\mathbf{s}: \mathbf{Z}, \mathbf{Z}:=\frac{\partial \chi^{\mathrm{C}}}{\partial \mathbf{H}}
$$

we can rewrite (5.7) as follows:

$$
\begin{aligned}
& -\int_{v}(\nabla \cdot \boldsymbol{\tau}+\mathbf{b}) \cdot \tilde{\mathbf{u}} \mathrm{d} v+\int_{v}(\mathbf{s}-\nabla \cdot \boldsymbol{\mu}): \tilde{\chi} \mathrm{d} \boldsymbol{v} \\
& \quad+\int_{\partial v}(\mathbf{n} \cdot \boldsymbol{\tau}-\mathbf{t}) \cdot \tilde{\mathbf{u}} \mathrm{da}+\int_{\partial v}(\mathbf{n} \cdot \boldsymbol{\mu}-\mathbf{m}): \tilde{\chi} \mathrm{da}=0 .
\end{aligned}
$$

Since the quantities within parentheses do not depend on the free virtual kinematic variables $\tilde{\mathbf{u}}$ and $\tilde{\chi}$, and (5.10) holds for arbitrary subdomain $v \subseteq V$, we can write as necessary and sufficient conditions:

$$
\left.\begin{array}{l}
\nabla \cdot \tau+\mathbf{b}=\mathbf{0} \text { in } v, \mathbf{n} \cdot \tau=\mathbf{t} \text { on } \partial v \\
\nabla \cdot \boldsymbol{\mu}-\mathbf{S}=\mathbf{0} \text { in } v, \mathbf{n} \cdot \boldsymbol{\mu}=\mathbf{m} \text { on } \partial v
\end{array}\right\}
$$

These are the field and boundary equilibrium equations for the macro- and microstructures, respectively. Eq. (5.11) differs from the classical equilibrium equations for a continuum only for the stress $\tau$ being not symmetric. The stress $\mathbf{s}$ represents the reaction stress of the microstructure against the continuum, evaluated within the microstructure tensor space, whereas the stress $\mathbf{s}: \mathbf{Z}$ is the same tensor quantity, but evaluated within the continuum tensor space. Remembering $(5.6)_{2,3}$, the relation $(5.11)_{3}$ can be rewritten as

$$
\frac{\partial \psi_{2}}{\partial \beta}-\nabla \cdot\left(\frac{\partial \psi_{2}}{\partial \kappa}\right)=0
$$

which is a tensor-valued second-order PDE relating the relative strain $\beta$ to $\chi$. The relative motion of the microstructure can be evaluated through the integration of (5.12), taking into account the related (standard) boundary conditions. These, remembering $(5.11)_{4}$, can be written as

$$
\boldsymbol{\chi}=\hat{\chi} \text { on } S_{\chi}, \quad \mathbf{n} \cdot \boldsymbol{\mu}=\hat{\mathbf{m}} \text { on } S_{m},
$$

where $S_{\chi} \cup S_{m}=S$, and $\hat{\chi}$, $\hat{\mathbf{m}}$ denote some specified surface functions.

The ER approach expounded above does not differ from the PVP one developed by Forest and Sievert [8] due to the lack of nonlocality sources. It is not the purpose of the present paper to address questions related to the solution of the above problem, for which the works of Mindlin [10], Kirchner and Steinmann [85], Forest [35] and Hirschberger and Steinmann [86] can be consulted.

\subsection{Local-type elastic material with elastically gradient microstructure}

In the previous subsection, the microstructure higherorder strain $\boldsymbol{\kappa}$ has been treated as independent of $\chi$ at the constitutive level. In this subsection, instead, we assume $\kappa \equiv \nabla \chi$ at the constitutive level, and thus $\chi$ constitutes a nonlocality-source strain. This implies that in view of the application of ER approach, the set of kinematically independent strain modes identifies with $(\varepsilon, \beta)$ and thus the stress power (5.3) reduces to

$$
\mathcal{W}=\sigma: \dot{\varepsilon}+\mathbf{s}: \dot{\beta},
$$

which remains still invariant under change of observer, whereas the free energy (5.1) takes on the form

$$
\psi=\psi_{1}(\varepsilon)+\psi_{2}(\beta, \nabla \chi) .
$$

Correspondingly, the Clausius-Duhem inequality is written as

$$
\sigma: \dot{\varepsilon}+\boldsymbol{s}: \beta-\dot{\psi}+\underbrace{\nabla \cdot(\mu: \dot{\chi})}_{P} \geq 0
$$

where we have introduced the residual $P=\nabla \cdot(\mu: \dot{\chi})$ to account for the nonlocality-source strain rate $\dot{\chi}$ (see (5.5) for comparison).

On expanding the time derivative of $\psi$ and the divergence operator, we have from (5.16), remembering (5.1),

$$
\begin{aligned}
\left(\boldsymbol{\sigma}-\frac{\partial \psi_{1}}{\partial \boldsymbol{\varepsilon}}\right): \dot{\varepsilon}+\left(s-\frac{\partial \psi_{2}}{\partial \boldsymbol{\beta}}\right):(\dot{\chi}-\mathbf{Z}: \dot{\mathbf{H}}) \\
+\nabla \cdot \boldsymbol{\mu}: \dot{\chi}+\left(\boldsymbol{\mu}-\frac{\partial \psi_{2}}{\partial \nabla \chi}\right): \nabla \dot{\chi} \geq 0
\end{aligned}
$$

where $\mathbf{Z}$ is given by (5.9). Since $\sigma$ is symmetric, we let $\dot{\boldsymbol{\varepsilon}}$ be replaced with $\dot{\mathbf{H}}$, and thus we can rewrite (5.17) as 


$$
\begin{gathered}
{\left[\boldsymbol{\sigma}-\frac{\partial \psi_{1}}{\partial \boldsymbol{\varepsilon}}-\left(\boldsymbol{s}-\frac{\partial \psi_{2}}{\partial \boldsymbol{\beta}}\right): \mathbf{Z}\right]: \dot{\mathbf{H}}+\left(\boldsymbol{s}-\frac{\partial \psi_{2}}{\partial \boldsymbol{\beta}}+\nabla \cdot \boldsymbol{\mu}\right): \dot{\chi}} \\
+\left(\boldsymbol{\mu}-\frac{\partial \psi_{2}}{\partial \nabla \chi}\right): \nabla \dot{\chi} \geq 0 .
\end{gathered}
$$

This inequality, due to the arbitrariness of the $\dot{\mathbf{H}}$ and $\dot{x}$ fields, yields the following state equations:

$$
\left.\begin{array}{l}
\boldsymbol{\sigma}=\frac{\partial \psi_{1}}{\partial \boldsymbol{\varepsilon}}-\operatorname{sym}(\nabla \cdot \boldsymbol{\mu}: \mathbf{Z}) \\
\mathbf{s}=\frac{\partial \psi_{2}}{\partial \beta}-\nabla \cdot \boldsymbol{\mu}, \boldsymbol{\mu}=\frac{\partial \psi_{2}}{\partial \nabla \chi}
\end{array}\right\}
$$

Equation (5.19) ${ }_{3}$ is a PDE system relating $\mu$ to $\chi$. The related higher-order boundary conditions can be easily derived by the insulation condition, but we skip this issue for brevity.

\subsection{Elastic-plastic material with gradient- type microstructure}

Here the continuum is considered as an elastic-plastic model exhibiting a total strain $\varepsilon=\varepsilon^{e}+\varepsilon^{p}$ and a (nonlinear) kinematic hardening described by the internal variable $\alpha$, a symmetric second-order tensor. The microstructure is an elastic-plastic model, featured by the microstrains $\chi=\varepsilon$ and by the strain-like pseudo-internal variable $a$, a symmetric second-order tensor, the convected part of which equals $\alpha$, i.e., $\mathrm{a}^{\mathrm{C}}=\alpha$. Continuing with the exploitation of the ER approach, the stress power and the free energy take on the forms:

$$
\mathcal{W}=\sigma: \dot{\varepsilon}, \quad \psi=\psi_{1}\left(\varepsilon-\varepsilon^{\mathrm{P}}, \alpha\right)+\psi_{2}(\mathbf{a}-\alpha, \nabla \mathbf{a}),
$$

where $\sigma$ is symmetric and thus $\mathcal{W}$ is invariant under change of observer. Since a constitutes a nonlocalitysource strain for the microstructure, the Clausius-Duhem inequality has to be cast in the (weak nonlocal) form:

$$
D=\sigma: \dot{\varepsilon}-\dot{\psi}+P \geq 0, \quad P=\nabla \cdot(\boldsymbol{\mu}: \dot{\mathbf{a}}),
$$

where $P$ is the pertinent ER, with $\mu=\left\{\mu_{k i j}\right\}$ being a thirdorder long-range stress, symmetric with respect to the index pair $(i, j)$.

Then, proceeding as usual, we can rewrite (5.21) as follows:

$$
\begin{aligned}
& D=\left(\boldsymbol{\sigma}-\frac{\partial \psi_{1}}{\partial \boldsymbol{\varepsilon}}\right): \dot{\varepsilon}-\frac{\partial \psi_{1}}{\partial \boldsymbol{\varepsilon}^{\mathrm{p}}}: \dot{\boldsymbol{\varepsilon}}^{\mathrm{p}}-\left(\frac{\partial \psi_{1}}{\partial \alpha}+\frac{\partial \psi_{2}}{\partial \alpha}\right): \dot{\alpha} \\
&+\left(\nabla \cdot \boldsymbol{\mu}-\frac{\partial \psi_{2}}{\partial \mathbf{a}}\right): \dot{\mathbf{a}}+\left(\boldsymbol{\mu}-\frac{\partial \psi_{2}}{\partial \nabla \mathbf{a}}\right): \nabla \dot{\mathbf{a}} \geq 0 .
\end{aligned}
$$

Assuming for simplicity a nondissipative microstructure and considering only deformation mechanisms with zero plastic strains $(\dot{\varepsilon}=\dot{\alpha}=\mathbf{0})$, inequality (5.22) simplifies as

$$
D=\left(\boldsymbol{\sigma}-\frac{\partial \psi_{1}}{\partial \boldsymbol{\varepsilon}}\right): \dot{\boldsymbol{\varepsilon}}+\left(\nabla \cdot \boldsymbol{\mu}-\frac{\partial \psi_{2}}{\partial \mathbf{a}}\right): \dot{\mathbf{a}}+\left(\boldsymbol{\mu}-\frac{\partial \psi_{2}}{\partial \nabla \mathbf{a}}\right): \nabla \dot{\mathbf{a}} \geq 0
$$

This, having to be valid for arbitrary $\dot{\varepsilon}$ and $\dot{\alpha}$ fields, gives

$$
\boldsymbol{\sigma}-\frac{\partial \psi_{1}}{\partial \boldsymbol{\varepsilon}}, \boldsymbol{\mu}=\frac{\partial \psi_{2}}{\partial \nabla \mathbf{a}}, \nabla \cdot \boldsymbol{\mu}=\frac{\partial \psi_{2}}{\partial \mathbf{a}}
$$

of which the first two relations are the elasticity laws of the continuum and the microstructure, respectively, whereas the third one is a restriction on $\alpha$.

Then, assuming that the latter relations continue to hold also for general elastic-plastic deformation mechanisms, inequality (5.22) reduces to:

$$
D=\sigma^{\mathrm{P}}: \dot{\varepsilon}^{\mathrm{P}}-\mathbf{X}: \dot{\alpha} \geq 0
$$

where

$$
\boldsymbol{\sigma}^{\mathrm{P}}:=\frac{\partial \psi_{1}}{\partial \boldsymbol{\varepsilon}^{\mathrm{P}}}=\boldsymbol{\sigma}
$$

and $\mathbf{X}$ is the total back stress tensor that, using (5.24), is given by

$$
\mathbf{X}:=\frac{\partial \psi_{1}}{\partial \alpha}+\frac{\partial \psi_{2}}{\partial \alpha}=\mathbf{X}_{0}-\nabla \mu, \mathbf{X}_{0}:=\frac{\partial \psi_{1}}{\partial \alpha} .
$$

Here, $\mathbf{X}_{0}$ is the standard lower-order back stress, and $\nabla \mu$ is its enhancement due to gradient effects entering through (5.24).

In the case of rate-independent and nonassociative plasticity, the evolution laws can be readily enforced by introducing a yield function $f$ and a potential function $g$ as, for instance,

$$
f=f_{1}(\boldsymbol{\sigma}-\mathbf{X})-\sigma_{0}, g=f_{1}(\boldsymbol{\sigma}-\mathbf{X})+f_{2}(\mathbf{X})
$$

where $\sigma_{0}$ is the yield strength, both $f_{1}$ and $f_{2}$ are convex functions, and in addition, $f_{2}$ is positive definite. Then, with the normality rule and a standard notation, the evolution laws can be cast in the form:

$$
\begin{gathered}
\dot{\varepsilon}^{\mathrm{P}}=\dot{\lambda} \frac{\partial g}{\partial \boldsymbol{\sigma}}=\dot{\lambda} \frac{\partial f}{\partial \boldsymbol{\sigma}}, \dot{\alpha}=-\dot{\lambda} \frac{\partial g}{\partial \mathbf{X}}=\dot{\boldsymbol{\varepsilon}}^{\mathrm{P}}-\dot{\lambda} \frac{\partial f_{2}}{\partial \mathbf{X}} \\
f(\boldsymbol{\sigma}-\mathbf{X}) \leq 0, \quad \dot{\lambda} \geq 0, \quad \dot{\lambda} f=0 .
\end{gathered}
$$

Following Lemaitre and Chaboche [73], it can be easily verified that any set of variables satisfying (5.29) complies 
with the dissipation inequality (5.25), since in fact we can write:

$$
D=\left(\boldsymbol{\sigma}: \frac{\partial g}{\partial \boldsymbol{\sigma}}+\mathbf{X}: \frac{\partial g}{\partial \mathbf{X}}\right) \dot{\lambda} \geq g \lambda=\left[\sigma_{0}+f_{2}(\mathbf{X})\right] \dot{\lambda} \geq 0
$$

with the equality sign holding only if $\dot{\lambda}=0$.

The above equations describe an elastic-plastic material with nonlinear kinematic hardening of gradient type, but with the gradient features indirectly described through the attached microstructure in a spirit quite similar to the so-called implicit gradient plasticity [79]. This means that the conditions in (5.29) are all local in nature and that, taking the microstrain a as known, their integration does not need any boundary conditions. These instead are required for the integration on the tensor-valued secondorder PDE:

$$
\frac{\partial \psi_{2}}{\partial \mathbf{a}}-\nabla \cdot\left(\frac{\partial \psi_{2}}{\partial \nabla \mathbf{a}}\right)=\mathbf{0}
$$

which is obtained from (5.24) $)_{2,3}$ by elimination of $\mu$. Eq. (5.31) relates the kinematic hardening variable $\alpha$. to the pseudo-internal variable a. Again, we can write out the latter boundary conditions using the insulation condition, for instance in its homogeneous form, i.e., recalling the expression of $P$ in (5.21) and the procedure used in Subsection 2.3 to arrive to (2.22):

$$
\mathbf{a}=\mathbf{0} \text { on } S_{\mathrm{h}}, \mathbf{n} \cdot \boldsymbol{\mu}=\mathbf{0} \text { on } S_{\mathrm{f}}
$$

For brevity, we do not elaborate this issue any further and note that the present formulation can accommodate the analogous one provided by Forest [35] for a more specialized constitutive model.

\subsection{Elastic Cosserat continua}

A Cosserat continuum can be considered as a special case of continuum with microstructure like the one addressed in Subsection 5.1, assuming that the microstrain $\chi$ is a skew-symmetric tensor, say $\chi=\omega=\left\{\omega_{i j}\right\}, \omega_{i j}=-\omega_{i j}$. This amounts to assuming that the microstructure (absolute) motion is a (local) rigid rotation, say $\phi$, independent of the analogous rotation of the continuum, say $\boldsymbol{v}=\frac{1}{2} \mathbf{e}: \mathbf{H}$. When $\chi=$ skwH everywhere in $V$, the Cosserat continuum specializes as a couple stress model $[1,3]$.

Cosserat continua have been the subject of numerous studies [see, e.g., Cosserat and Cosserat [6], Besdo [7], Forest and Sievert [8] and references therein], but as in Subsection 5.1, we present here some developments that are believed to be useful for a better understanding of the (herein applied) ER-based theory. For this purpose, let us first reconsider the stress power (5.3) using the position $\chi=\omega$. We can thus write:

$$
\mathcal{W}=\underbrace{[\sigma: \dot{\varepsilon}+\mathrm{s}:(\dot{\omega}-\mathrm{skw} \dot{\mathbf{H}})]}_{w_{1}}+\underbrace{\mu: \nabla \dot{\omega}}_{\mathcal{W}_{2}}
$$

where $\boldsymbol{\sigma}=\left\{\sigma_{i j}\right.$ is symmetric, whereas $\mathbf{s}=\left\{\mathrm{s}_{\mathrm{ij}}\right\}$ and $\boldsymbol{\mu}=\left\{\mu_{k i j}\right\}$ are skew-symmetric [ $\boldsymbol{\mu}$ with respect to the last index pair $(i, j)]$. The sum of the first two addends of (5.33) (within square brackets) can be transformed as

$$
\mathcal{W}_{1}:=(\boldsymbol{\sigma}-\mathbf{s}): \dot{\mathbf{H}}+\mathbf{s}: \dot{\omega}=\mathbf{T}: \dot{\mathbf{H}}+\mathbf{s}: \dot{\omega}
$$

where $\mathbf{T}:=\boldsymbol{\sigma}$-s; the third addend of (5.33) can in turn be rewritten, using the relation $\dot{\omega}=-\mathbf{e} \cdot \dot{\boldsymbol{\phi}}$, as

$$
\mathcal{W}_{2}:=\mu: \nabla(-\mathbf{e} \cdot \dot{\phi})=(-\mu: \mathbf{e}): \nabla \dot{\phi}=\mathbf{m}: \dot{\kappa}
$$

where $\mathbf{m}:=-\mu: \mathbf{e}$ denotes the couple stress tensor and $\boldsymbol{\kappa}:=\nabla \boldsymbol{\phi}$ is the curvature tensor. Considering that $\nabla \dot{\boldsymbol{\omega}} \rightarrow \nabla \dot{\mathbf{W}}$ under change of observer and that therefore $\mathcal{W}_{2}$ is invariant correspondingly, the analogous requisite of $\mathcal{W}_{1}+\mathcal{W}_{2}$ demands that $\mathcal{W}_{1}$ also satisfies the latter requisite. For a change of observer, we have $\dot{\mathbf{H}} \rightarrow \dot{\mathbf{W}}$ and $\dot{\boldsymbol{\omega}} \rightarrow \dot{\mathbf{W}}$; thus, the condition $(\mathbf{T}+s): \dot{\mathbf{W}}=0, \forall \dot{\mathbf{W}}$ must be satisfied. This implies that $\operatorname{skw}(\mathbf{T}+\mathbf{s})=0$, i.e., skw $\mathbf{s} \equiv \mathbf{s}$ identifies with the negative skew-symmetric part of $\mathbf{T}$. With this result in mind, we can write:

$$
\mathcal{W}_{1}=\mathbf{T}: \dot{\mathbf{H}}-\mathrm{skwT}: \dot{\omega}=\mathbf{T}:(\dot{\mathbf{H}}-\dot{\omega})
$$

hence the stress power (5.32) takes on the form

$$
\mathcal{W}=\mathbf{T}: \dot{\mathbf{c}}+\mathbf{m}: \dot{\boldsymbol{\kappa}},
$$

where the strain tensor

$$
\mathbf{c}:=\mathbf{H}-\omega=\nabla \mathbf{u}+\mathbf{e} \phi
$$

denotes the so-called Cosserat strain.

Next, noting that

$$
\boldsymbol{\varepsilon}=\operatorname{sym} \mathbf{c}, \beta=\omega \text {-skw } \mathbf{H}=\text {-skw } \mathbf{c}, \nabla \boldsymbol{\kappa}=\nabla(-\mathbf{e} \cdot \boldsymbol{\phi})=-\mathbf{e} \cdot \boldsymbol{\kappa}^{T},
$$

we can recognize that the free energy (5.2) is equivalent to

$$
\psi=\psi(\mathbf{c}, \boldsymbol{\kappa}) .
$$

The material exhibits kinematically independent deformation modes $(\mathbf{c}, \boldsymbol{\kappa})$, controlled by the degrees of freedom $(\mathbf{u}, \phi)$. Since no ER $P$ is required due to the absence of any nonlocality-source strain, the 
Clausius-Duhem inequality (2.10) takes the standard format:

$$
\mathbf{T}: \dot{\mathbf{c}}+\mathbf{m}: \dot{\boldsymbol{\kappa}}-\dot{\psi}(\mathbf{c}, \boldsymbol{\kappa}) \geq 0
$$

from which we readily obtain:

$$
\mathbf{T}=\frac{\partial \psi}{\partial \mathbf{c}}, \mathbf{m}=\frac{\partial \psi}{\partial \kappa}
$$

The equilibrium equations can then be derived by the (standard) PVP, namely

$$
\begin{aligned}
\int_{v}[\mathbf{T} & :(\nabla \tilde{\mathbf{u}}+\mathbf{e} \cdot \tilde{\boldsymbol{\phi}})+\mathbf{m}: \nabla \tilde{\boldsymbol{\phi}}] \mathrm{d} v=\int_{v}(\mathbf{b} \cdot \tilde{\mathbf{u}}+\mathbf{f} \cdot \tilde{\boldsymbol{\phi}}) \mathrm{d} v \\
& +\int_{\partial v}(\mathbf{t} \cdot \tilde{\mathbf{u}}+\mathbf{p} \cdot \tilde{\boldsymbol{\phi}}) \mathrm{d} a
\end{aligned}
$$

where $\mathbf{f}$ and $\mathbf{p}$ denote body and surface couple stresses. Then, through a straightforward procedure, the equilibrium equations can be written as:

$$
\begin{gathered}
\nabla \cdot \mathbf{T}+\mathbf{b}=0 \text { in } v, \mathbf{n} \cdot \mathbf{T}=\mathbf{t} \text { on } \partial v \\
\nabla \cdot \mathbf{m}-\mathbf{T}: \mathbf{e}+\mathbf{f}=\mathbf{0} \text { in } v, \mathbf{n} \cdot \mathbf{m}=\mathbf{p} \text { on } \partial v .
\end{gathered}
$$

The above ER-based procedure coincides in most part with the one by Forest and Aifantis [36]. Indeed, in the absence of any nonlocality-source strain, the ER-based procedure identifies with the PVP-based one.

\subsection{Elastic-plastic Cosserat continua with strengthening effects}

Let the Cosserat material of the previous subsection be elastic-plastic and let the additive strain decomposition hold, namely

$$
\mathbf{C}=\mathbf{c}^{\mathrm{e}}+\mathbf{c}^{\mathrm{p}}, \boldsymbol{\kappa}=\boldsymbol{\kappa}^{\mathrm{e}}+\boldsymbol{\kappa}^{\mathrm{p}} .
$$

Continuing with the application of the ER approach, let the free energy (5.40) be replaced with one of the form

$$
\psi=\psi_{1}\left(\mathbf{c}-\mathbf{c}^{\mathrm{p}}, \boldsymbol{\kappa}-\boldsymbol{\kappa}^{\mathrm{p}}\right)+\psi_{2}(\gamma, \nabla \gamma)
$$

where $\gamma$ is a scalar pseudo-internal variable having the meaning of effective plastic strain. Both $\psi_{1}$ and $\psi_{2}$ are convex homogeneous functions, $\psi_{1}$ of degree $d_{1} \geq 2, \psi_{2}$ of degree $d_{2}=1$. The material is gradient type and induces size effects on the yield strength, or strengthening effects $[57,58]$, but is local-type for the rest. Hence, the Clausius-Duhem inequality (2.10) in isothermal conditions reads

$$
D=\mathbf{T}: \dot{\mathbf{c}}+\mathbf{m}: \dot{\boldsymbol{\kappa}}-\dot{\psi}+P \geq 0, \quad P=\nabla \cdot(\mathbf{R} \dot{\gamma})
$$

where $\mathbf{R}$ is some stress-like vector associated to $\dot{\gamma}$. Let us expand the time derivative of $\psi$ and the divergence operator of $P$ and let us then rewrite (5.48) as

$$
\begin{aligned}
\mathbf{T}-\frac{\partial \psi_{1}}{\partial \mathbf{c}}: \dot{\mathbf{c}} & +\left(\mathbf{m}-\frac{\partial \psi_{1}}{\partial \boldsymbol{\kappa}}\right): \dot{\boldsymbol{\kappa}}-\frac{\partial \psi_{1}}{\partial \mathbf{c}^{\mathrm{P}}}: \dot{\mathbf{c}}^{\mathrm{P}}-\frac{\partial \psi_{1}}{\partial \boldsymbol{\kappa}^{\mathrm{P}}}: \dot{\boldsymbol{\kappa}}^{\mathrm{P}} \\
& +\left(\nabla \cdot \mathbf{R}-\frac{\partial \psi_{2}}{\partial \gamma}\right) \dot{\gamma}+\left(\mathbf{R}-\frac{\partial \psi_{2}}{\partial \nabla \gamma}\right) \cdot \nabla \dot{\gamma} \geq 0 .
\end{aligned}
$$

This inequality gives the elasticity laws, formally coincident with (5.42), i.e.,

$$
\mathbf{T}=\frac{\partial \psi_{1}}{\partial \mathbf{c}}, \quad \mathbf{m}=\frac{\partial \psi_{1}}{\partial \boldsymbol{\kappa}}
$$

Then, assuming the constitutive equation

$$
\mathbf{R}:=\frac{\partial \psi_{2}}{\partial \nabla \gamma}
$$

and with the positions

$$
\mathbf{T}^{\mathrm{P}}:=\frac{\partial \psi_{1}}{\partial \mathbf{c}^{\mathrm{P}}}=\mathbf{T}, \quad \mathbf{m}^{\mathrm{P}}:=-\frac{\partial \psi_{1}}{\partial \boldsymbol{\kappa}^{\mathbf{P}}}=\mathbf{m}
$$

and

$$
Y:=\frac{\partial \psi_{2}}{\partial \gamma}-\nabla \cdot\left(\frac{\partial \psi_{2}}{\partial \nabla \gamma}\right)
$$

the dissipation inequality takes on the final form

$$
D=\mathbf{T}^{\mathrm{P}}: \dot{\mathbf{c}}^{\mathrm{P}}+\mathbf{m}^{\mathrm{P}}: \dot{\boldsymbol{\kappa}}^{\mathrm{P}}-Y \dot{p} \geq 0 .
$$

The scalar $Y$ is a degree-zero homogeneous function of $\gamma$ and $\nabla \gamma$; it thus represents some strengthening effects in the sense that it is the increase of the initial yield strength $\sigma_{0}$ caused by the deformation process; hence, the actual yield strength is expressed as

$$
\sigma_{y}=\sigma_{0}+Y
$$

$Y$, here called strengthening stress, is related to $\gamma$ by (5.53), which is a second-order PDE. For a given $Y$ field, (5.53) can be integrated to obtain the related $\gamma$ field. The boundary conditions required for this purpose can be established by means of the insulation condition. This, in its homogeneous form, remembering the form of $P$ in (5.48), gives:

$$
\int_{V} P \mathrm{~d} v=\int_{S} \mathbf{n} \cdot \mathbf{R} \dot{\gamma} \mathrm{d} a=0
$$

and therefore the boundary conditions can be enforced as follows: 


$$
\dot{\gamma}=\gamma=0 \text { on } S_{\mathrm{h}} \quad \mathbf{n} \cdot \mathbf{R}=0 \text { on } S_{\mathrm{f}}
$$

For a rate-independent associative model, the evolution laws can be established in a standard way by writing, for instance,

$$
\left.\begin{array}{l}
f=f_{0}\left(\mathbf{T}, \frac{1}{l} \mathbf{m}\right)-\sigma_{y} \leq 0, \quad \dot{\lambda} \geq 0, \quad \dot{\lambda} f=0 \\
\dot{\mathbf{c}}^{\mathrm{P}}=\dot{\lambda} \frac{\partial f_{0}}{\partial \mathbf{T}}, \quad \dot{\kappa}^{\mathrm{P}}=\dot{\lambda} \frac{\partial f_{0}}{\partial\left(\frac{1}{l} \mathbf{m}\right)} \frac{1}{l}, \dot{\gamma}=\dot{\lambda}
\end{array}\right\}
$$

where $l$ denotes an internal length scale parameter and $f_{0}$ is a convex degree-one homogeneous function. It is

$$
\dot{\gamma}=\sqrt{\left\|\dot{\mathbf{c}}^{\mathrm{P}}\right\|^{2}+l^{2}\left\|\dot{\boldsymbol{\kappa}}^{\mathrm{P}}\right\|^{2}}
$$

A simple choice for $\psi_{2}$ is

$$
\psi_{2}=\sigma_{0}[\varphi(\gamma, \nabla \gamma)-p], \varphi=\sqrt{\gamma^{2}+l^{2}\|\nabla \gamma\|^{2}}
$$

which by (5.53) and (5.55) gives the differential equation

$$
\frac{\sigma_{\mathrm{y}}}{\sigma_{0}}=\frac{\gamma}{\varphi}-l_{2} \nabla \cdot\left(\frac{\nabla \gamma}{\varphi}\right)
$$

The consistency condition $\dot{f}=0$ provides a nonlinear second-order PDE for the evaluation of $\dot{\gamma}=\dot{\lambda}$, which can be verified to be of the form

$$
\mathcal{L}(\dot{\gamma}, \nabla \dot{\gamma}, \nabla \dot{\gamma})=\mathbf{A}_{\mathrm{c}}: \dot{\mathbf{c}}+\mathbf{A}_{\kappa}: \dot{\boldsymbol{\kappa}},
$$

where $\mathcal{L}$ is a linear second-order differential operator, the coefficients of which, together with $\mathbf{A}_{c}$ and $\mathbf{A}_{k}$, depend on the current material state. Eq. (5.62) can be integrated taking into account the higher-order boundary conditions (5.57) written in rate form [with the proviso that at the points of the (moving) elastic-plastic interface, if any, both Dirichlet and Neumann boundary conditions must be enforced].

The continuum tangent stiffness matrices can be obtained starting from the elasticity laws (5.50) written in rate form, say,

$$
\dot{\mathbf{T}}=\mathbf{L}:\left(\dot{\mathbf{c}}-\dot{\mathbf{c}}^{\mathrm{P}}\right), \dot{\mathbf{m}}=\mathbf{M}:\left(\dot{\boldsymbol{\kappa}}-\dot{\boldsymbol{\kappa}}^{\mathrm{P}}\right)
$$

from where we can obtain, by $(5.58)_{4,5}$

$$
\begin{aligned}
& \mathbf{K}_{\mathrm{c}}:=\frac{\partial \dot{\mathbf{T}}}{\partial \dot{\mathbf{c}}}=\mathbf{L}-\mathbf{L}: \frac{\partial f_{0}}{\partial \mathbf{T}} \otimes \mathbf{J}_{\mathrm{c}} \\
& \mathbf{K}_{\kappa}:=\frac{\partial \dot{\mathbf{m}}}{\dot{\partial \dot{\boldsymbol{\kappa}}}}=\mathbf{M}-\mathbf{M}: \frac{\partial f_{0}}{\partial \mathbf{m}} \otimes \mathbf{J}_{\kappa} .
\end{aligned}
$$

The matrices $\mathbf{J}_{c}$ and $\mathbf{J}_{\kappa}$, characterizing the plastic sensitivity of the structural model to the total strain rates, are given by

$$
\mathbf{J}_{\mathrm{c}}:=\frac{\partial \dot{\gamma}}{\partial \dot{\mathbf{c}}}, \quad \mathbf{J}_{\kappa}:=\frac{\partial \dot{\gamma}}{\partial \dot{\boldsymbol{\kappa}}}
$$

The latter matrices can be formally built as solutions of the PDE (5.62), namely.

$$
\mathcal{L}\left(\mathbf{J}_{\alpha}, \nabla \mathbf{J}_{\alpha}, \Delta \mathbf{J}_{\alpha}\right)=\mathbf{A}_{\alpha}, \forall_{\alpha}=c, \kappa .
$$

The higher-order boundary conditions that accompany (5.66) can be specified making reference to (5.57). Since by (5.51) and (5.60) it is

$$
\mathbf{n} \cdot \mathbf{R}=l^{2} \sigma_{0} \partial_{n} \gamma / \varphi=0 \rightarrow \partial_{n} \gamma=0,
$$

the mentioned boundary conditions can be written

$$
\dot{\gamma}=0 \text { on } S_{\mathrm{h}}, \partial_{n} \dot{\gamma}=0 \text { on } S_{\mathrm{f}} .
$$

These, since the $\dot{\gamma}$ response is parametrically dependent on the total strain rates, $\dot{\mathbf{c}}$ and $\dot{\kappa}$, by (5.62) prove to be equivalent to:

$$
\mathbf{J}_{\alpha}=\mathbf{0} \text { on } S_{\mathrm{h}}, \partial_{n} \mathbf{J}_{\alpha}=\mathbf{0} \text { on } S_{\mathrm{f}}, \forall \alpha=\mathrm{c}, \kappa
$$

which are the boundary conditions accompanying the PDEs (5.66).

The numerical implementation of the above procedure for particular simpler situations (as plane stress and plane strain conditions) is under study.

\section{Conclusions}

Generalized continua as nonpolar and polar, or micromorphic, materials that exhibit a gradient-type behavior have been addressed by means of a (weak) nonlocal approach stemming from the so-called energy residual (ER)-based theory of strain gradient plasticity [27, 52, 55, $58,59]$, cast in a form suitable to cope with a wider spectrum of gradient models of continuum mechanics. A main feature of the latter approach is that the thermodynamics principle of the local actions does not hold; hence, the first thermodynamics principle written in pointwise form requires the addition of an extra term accounting for the long-range energy interchanges. This extra term, called "energy residual", has been shown to be quite different in nature from the so-called "extra entropy flux" by Maugin [13]. Another main feature of the proposed approach consists in maintaining the formulation of the constitutive equations - including those shaped as PDEs with associated higher-order boundary conditions - within the strict 
confines of (nonlocal) thermodynamics similarly to the analogous classical equations, leaving out the PVP. The latter saves its standard format and intervenes only at the global structural level when the relevant degrees of freedom (displacements) enter into play.

It is the authors' belief that the proposed approach constitutes a valid alternative to the PVP-based approach and possesses some advantages such as the following:

- The ER approach evaluates the constitutive equations of a gradient material (including generalized continua), shaped as PDEs with related higher-order boundary conditions, in a direct way by means of the (weak nonlocal) Clausius-Duhem inequality, without the necessity to invoke the PVP.
- In doing that, the ER approach considers just one (higher-order) microstress for every strain gradient, to which it recognizes the physical meaning of longrange stress operating in parallel to the short-range Cauchy stress.

- The insulation condition, a typical ingredient of the ER approach, constitutes a means to evaluate the spatial extent within which the material particles influence one another at the constitutive level; it thus offers an effective way to determine the higher-order boundary conditions completing the differential-type constitutive equation formulation for a gradient material.

- The ER approach applies as well to materials of true nonlocal (integral) type [62, 63, 87].

\section{References}

[1] Mindlin RD, Tiersten, HF. Arch. Ration. Mech. Anal. 1962, 11, 415-488.

[2] Toupin RA. Arch. Ration. Mech. Anal. 1962, 11, 385-414.

[3] Toupin RA. Arch. Ration. Mech. Anal. 1964, 17, 85-112.

[4] Mindlin RD. Int. J. Solids Struct. 1965, 1, 417-438.

[5] Mindlin RD, Eshel NN. Int. J. Solids Struct. 1968, 4, 109-124.

[6] Cosserat E, Cosserat F. Théorie des Corps Déformable, Hermann et Fils: Paris, 1909.

[7] Besdo B. Acta Mech. 1974, 20, 105-131.

[8] Forest S, Sievert R. Acta Mech. 2003, 160, 71-111.

[9] Eringen AC, Suhubi ES. Int. J. Eng. Sci. 1964, 2, 189-203.

[10] Mindlin RD. Arch. Ration. Mech. Anal. 1964, 16, 51-78.

[11] Dillon OW, Kratochvil J. Int. J. Solids Struct. 1970, 6, 1513-1533.

[12] Sidoroff F. Mech. Res. Commun. 1975, 2, 73-77.

[13] Maugin GA. J. Non-Equilib. Thermodyn. 1990, 15, 173-192.

[14] Fleck NA, Hutchinson JW. J. Mech. Phys. Solids 1993, 41, 1825-1857.

[15] Aifantis EC. J. Eng. Mater. Technol. 1984, 106, 326-330.

[16] Aifantis EC. Phase Transformation in Solids, Tsakalakos T, Ed. North Holland, Amsterdam, 1984, pp. 37-49.

[17] Aifantis EC. Int. J. Eng. Sci. 1984, 22, 961-968.

[18] Aifantis EC. Int. J. Plast. 1987, 3, 211-248.

[19] Aifantis EC. Int. J. Fract. 1999, 95, 299-314.

[20] Engelen RAB, Fleck NA, Peerlings RHJ, Geers MGD. Int. J. Solids Struct. 2006, 43, 1857-1877.

[21] Bardella L. Int. J. Eng. Sci. 2010, 48, 550-560.

[22] Mühlhaus H-B, Aifantis EC. Acta Mech. 1991, 89, 217-231.

[23] Aifantis EC. Int. J. Eng. Sci. 1992, 30, 1279-1299.

[24] Aifantis KE, Willis JR. J. Mech. Phys. Solids 2005, 53, 1047-1070.

[25] Aifantis KE, Soer WA, De Hosson JTh, Willis JR. Acta Mater. 2006, 54, 5077-5085.

[26] Fredriksson P, Gudmundson P. J. Mech. Phys. Solids 2007, 55, 939-955.

[27] Polizzotto C. Int. J. Solids Struct. 2009, 46, 1685-1694.

[28] Fleck NA, Hutchinson JW. Adv. Appl. Mech. 1997, 33, 295-361.

[29] Aifantis EC. Mech. Mater. 2003, 35, 259-280.

[30] Kuroda M, Tvergaard V. Int. J. Plast. 2010, 26, 507-515.
[31] Germain P. J. Méc. 1973a, 12, 235-274.

[32] Germain P. SIAM J. Appl. Math. 1973b, 25, 556-575.

[33] Maugin GA. Acta Mech. 1980, 35, 1-70.

[34] Maugin GA, Muschik W. J. Non-Equilib. Thermodyn. 1994, 19, 217-249.

[35] Forest S. J. Eng. Mech. Div., Am. Soc. Civ. Eng. 2009, 135, 117-131.

[36] Forest S, Aifantis EC. Int. J. Solids Struct. 2010, 47, 3367-3376.

[37] Forest S, Cardona J-M, Sievert R. In Continuum Thermodynamics, Maugin GA, Drouot F, Sidoroff F, Eds. Kluwer Academic Publ.: Dordrecht, 2000, pp. 163-176.

[38] Gao H, Huang Y, Nix WD, Hutchinson JW. J. Mech. Phys. Solids 1999, 47, 1239-1263.

[39] Acharya A, Bassani JL. J. Mech. Phys. Solids 2000, 48, 1565-1595.

[40] Fleck NA, Hutchinson JW. J. Mech. Phys. Solids 2001, 49, 2245-2271.

[41] Liebe T, Steinmann P. Int. J. Numer. Methods Eng. 2001, 51, 1437-1467.

[42] Gurtin ME. J. Mech. Phys. Solids 2000, 48, 989-1036.

[43] Gurtin ME. J. Mech. Phys. Solids 2002, 50, 5-32.

[44] Gurtin ME. Int. J. Plast. 2003, 19, 47-90.

[45] Gurtin ME. J. Mech. Phys. Solids 2004, 52, 2545-2568.

[46] Gudmundson P. J. Mech. Phys. Solids 2004, 52, 1379-1406.

[47] Anand L, Gurtin ME, Lele SR, Getting C. J. Mech. Phys. Solids 2005, 53, 1789-1826.

[48] Fleck NA, Willis JR. J. Mech. Phys. Solids 2009, 57, 161-177.

[49] Fleck NA, Willis JR. J. Mech. Phys. Solids 2009, 57, 1045-1057.

[50] Voyiadjis GZ, Deliktas B. Int. J. Plast. 2009, 25, 1997-2024.

[51] Voyiadjis GZ, Pekmezi G, Deliktas B. Int. J. Plast. 2010, 26, $1335-1336$.

[52] Polizzotto C, Borino G. Eur. J. Mech. A/Solids 1998, 17, 741-761.

[53] Polizzotto C. Int. J. Solids Struct. 2003, 40, 7399-7423.

[54] Polizzotto C. Eur. J. Mech. A/Solids 2003, 22, 651-668.

[55] Polizzotto C. Eur. J. Mech. A/Solids 2007, 26, 189-211.

[56] Polizzotto C. Int. J. Plast. 2009, 25, 2169-2180.

[57] Polizzotto C. Int. J. Solids Struct. 2010, 47, 100-112. 
[58] Polizzotto C. Int. J. Plast. 2011, 27, 388-413.

[59] Borino G, Polizzotto C. Modell. Simul. Mater. Sci. Eng. 2007, 15, 23-35.

[60] Borino G, Failla B, Parrinello F. Int. J. Solids Struct. 2003, 40, 3621-3645.

[61] Edelen DGB, Laws N. Arch. Ratio. Mech. Anal. 1971, 43, 24-35.

[62] Polizzotto C. Int. J. Solids Struct. 2001, 38, 7359-7380.

[63] Polizzotto C, Fuschi P, Pisano AA. Eur. J. Mech. A/Solids 2006, 25, 308-333.

[64] Eringen AC, Edelen DGB. Int. J. Eng. Sci. 1972, 10, 233-248.

[65] Dunn JE, Serrin J. Arch. Ration. Mech. Anal. v, 88, 95-133.

[66] Casal P, Gouin E. C. R. Acad., Ser. II: Mec. Phys. Chim. Sci. Terre Univers 1998, 306, 99-104.

[67] Müller I. Thermodynamics. Pitman: London, 1985.

[68] Truesdell C, Noll W. In Encyclopedia of Physics, Volume III/3, Flügge S. Ed. Springer-Verlag: Berlin, Heidelberg, New York, 1965.

[69] Maugin GA. The Thermodynamics of Nonlinear Irreversible Behaviors, World Scientific Publishing Co.: Singapore, 1999.

[70] Colemann BD, Gurtin ME. J. Chem. Phys. 1967, 47, 597-613.

[71] Malvern LE. Introduction to Mechanics of a Continuous Medium, Prentice-Hall, Inc.: Englewood Cliffs, NJ, 1969.

[72] Germain P, Nguyen QS, Suquet P. J. Appl. Mech. 1983, 50, 731-742.

[73] Lemaitre J, Chaboche J-L. Mechanics of Solid Materials, University Press: Cambridge, UK, 1990.
[74] Polizzotto C. Int. J. Solids Struct. 2012, 49, 2121-2137.

[75] Gurtin ME, Murdock I. Arch. Ration. Mech. Anal. 1975, 57, 291-323.

[76] Green AE, Rivlin RS. Arch. Ration. Mech. Anal. 1964, 16, 325-353.

[77] Lazar M, Maugin GA, Aifantis EC. Int. J. Solids Struct. 2006, 43, 1787-1817.

[78] Ashby MF. Philos. Mag. 1970, 21, 399-424.

[79] Engelen RAB, Geers MGD, Baaijens FPT. Int. J. Plast. 2003, 19, 403-433.

[80] Asaro RJ. Adv. Appl. Mech. 1983, 23, 1-115.

[81] Forest S, Sievert R, Aifantis EC. J. Mech. Behav. Mater. 2002, 13, 219-232.

[82] Nicola L, van der Giessen E, Gurtin ME. J. Mech. Phys. Solids 2005, 53, 1280-1294.

[83] Gurtin ME, Anand L, Lele SP. Int. J. Solids Struct. 2007, 55, 1853-1878.

[84] Gurtin ME, Needleman A. J. Mech. Phys. Solids 2005, 53, $1-31$.

[85] Kirchner N, Steinmann P. Philos. Mag. 2005, 85, 3875-3895.

[86] Hirschberger B, Steinmann P. J. Eng. Mech. Div. Am. Soc. Civ. Eng. 2009, 135, 156-170.

[87] Polizzotto C, Borino G. In: Encyclopedia of Thermal Stresses, Hetnarski RB, Ed. Springer-Verlag: Heidelberg, in press. 